\title{
ON MULTIVARIATE DISCRETE MOMENT PROBLEMS: GENERALIZATION OF THE BIVARIATE MIN ALGORITHM FOR HIGHER DIMENSIONS*
}

\author{
GERGELY MÁDI-NAGY ${ }^{\dagger}$
}

\begin{abstract}
The objective of the multivariate discrete moment problem (MDMP) is to find the minimum and/or maximum of the expected value of a function of a random vector with a discrete finite support where the probability distribution is unknown, but some of the moments are given. The moments may be binomial, power, or of a more general type. The MDMP can be formulated as a linear programming problem with a very ill-conditioned coefficient matrix. Hence, the LP problem can be solved with difficulty or cannot be solved at all. The central results of the field of the MDMP concern the structure of the dual feasible bases. These bases, on one hand, provide us with bounds without any numerical difficulties. On the other hand, they can be used as an initial basis of the dual simplex method. That results in shorter running time and better numerical stability because the first phase can be skipped. This paper introduces a new type of MDMP, where the bivariate moments up to a certain order $m$ consisting of the first variable and further univariate moments up to the order $m_{j}, j=1, \ldots, s$, are given. Then we generalize the bivariate Min Algorithm of Mádi-Nagy and Prékopa [Math. Oper. Res., 29 (2004), pp. 229-258] for higher dimensions, which gives numerous dual feasible bases of the MDMP. By the aid of this, on one hand, we can give useful bounds for MDMPs with higher dimensional random vectors even if the usual solvers cannot give acceptable results. On the other hand, applying our algorithm for the binomial MDMP, we can give better bounds for probabilities of Boolean functions of event sequences than the recent bounds in the literature. These results are illustrated by numerical examples.
\end{abstract}

Key words. discrete moment problem, multivariate Lagrange interpolation, linear programming, expectation bounds, probability bounds

AMS subject classifications. 62H99, 90C05, 65D05

DOI. $10.1137 / 070705878$

\section{Introduction.}

1.1. Moment problems. The classical moment problem was introduced in 1894-1895 by Stieltjes [38] (see Kjeldsen [18]). The problem is about trying to invert the mapping that takes a measure $P$ on $I \subset \mathbb{R}$ to the sequences of moments $\mu_{k}$, $k=1,2,3, \ldots$, where the $k$ th, so-called power, moments are defined as

$$
\mu_{k}=\int_{I} z^{k} d P
$$

In what follows, we will use the notation $\mu_{0}$ for $k=0$, but we know $\mu_{0}=1$. More general moments than the power moments can be defined if we consider the sequence of functions $u_{k}(z), k=0,1,2, \ldots$, which are assumed to be measurable and integrable functions on $I$. Then the generalized moment sequence with respect to $\left\{u_{k}(z)\right\}$ is

$$
\int_{I} u_{k}(z) d P, \quad k=0,1, \ldots
$$

*Received by the editors October 17, 2007; accepted for publication (in revised form) October 12, 2008; published electronically February 11, 2009. This research was partially supported by OTKA grants F-046309 and T-047340 in Hungary.

http://www.siam.org/journals/siopt/19-4/70587.html

${ }^{\dagger}$ Mathematical Institute, Budapest University of Technology and Economics, Müegyetem rakpart 1-3, Budapest 1111, Hungary (gnagy@math.bme.hu). 
Sometimes the moment problem is based on these general moments, but typically power moments are used. The questions of the classical moment problem can be the following. Given a sequence of numbers $\mu_{k}, k=1,2,3, \ldots$,

(a) does there exist a probability measure on $I$ with the moments $\mu_{k}, k=$ $1,2,3, \ldots$ ?

(b) is this probability measure uniquely determined by the moments $\mu_{k}, k=$ $1,2,3, \ldots$ ?

(c) how can one describe all probability measures on $I$ with the moments $\mu_{k}$, $k=1,2,3, \ldots$ ?

Depending on the set $I$, classical results can be found in Hamburger [12], [13], Hausdorff [14], [15], and Riesz [36]. Akhiezer [1] gives a comprehensive survey of that field.

The truncated variation of the classical moment problem studies the properties of measures with fixed first $k$ moments (for a finite $k$ ) or with finite set of moments. Beside the classical questions (existence, uniqueness of the measure $P$ ) the following bounding problem arises. Given the moments $\mu_{k}, k=1, \ldots, m$, or any finite collection of the moments, what are the possible lower and upper bounds for

$$
\int_{I} f(z) d P
$$

where $f$ is a given real function on $I$ and $P$ is unknown, but $P$ has to have the given moments? Let $X$ be a random variable with support $I$. Assume that the expected values of $X^{k}, k=1, \ldots, m$, are given finite values, but the distribution of $X$ is not known. Then the bounding moment problem can be formulated as

$$
\inf (\sup ) E[f(X)]=\int_{I} f(z) d P
$$

subject to

$$
E\left[X^{k}\right]=\int_{I} z^{k} d P=\mu_{k}, \quad k=0,1, \ldots, m,
$$

where the probability measure $P$ is unknown, and $I, f, \mu_{k}, k=0,1, \ldots, m$, are given. Bounding problems related to moments were first considered by Bienaymé [2], Chebyshev [6], [7], and Markov [23]. The bounding moment problem frequently appears in the literature as Chebyshev-type inequalities. A good summary of these results can be found in Krein and Nudelman [19]. For historical background, see Kjeldsen [18] and Prékopa and Alexe [33].

At the end of the 1980s, Prékopa [25], [26], [27] and Samuels and Studden [37] independently introduced and studied the univariate discrete moment problem (DMP), where $I=\left\{z_{0}, z_{1}, \ldots, z_{n}\right\}$ is a discrete finite set. Samuels and Studden use the classical approach and determine the solutions in closed form whenever possible; however, their method is applicable only to small size problems. Prékopa invented a linear programming methodology, presented briefly below. It turns out that in the special case of the DMP, linear programming techniques provide us with more general and simpler algorithmic solutions than the classical ones. Moreover, it allows for an efficient solution of large size moment problems as well as for finding closed form sharp bounds.

The DMP has (at least) three useful properties. The first one is that it uses the discrete property of the support $I$ beside the moment information. Hence, it can give tighter bounds than the classical moment problems. The second one is that the 
DMP can be formulated as a linear programming (LP) problem. Let us designate the (unknown) probability distribution of $X$ in the following way:

$$
p_{i}=P\left(X=z_{i}\right), \quad i=0,1, \ldots, n,
$$

and let $f\left(z_{i}\right)=f_{i}$. Then

$$
E[f(X)]=f_{0} p_{0}+f_{1} p_{1}+\cdots+f_{n} p_{n}
$$

and

$$
E\left[X^{k}\right]=z_{0}^{k} p_{0}+z_{1}^{k} p_{1}+\cdots+z_{n}^{k} p_{n}, \quad k=0,1, \ldots, m .
$$

The LP corresponding to the DMP is

$$
\begin{array}{rrrrrl}
\min (\max ) & f_{0} p_{0} & +f_{1} p_{1} & +\cdots & +f_{n} p_{n} & \\
\text { subject to } & & & \multicolumn{1}{l}{} & \\
& p_{0} & +p_{1} & +\cdots & +p_{n} & =\mu_{0}(=1), \\
& z_{0} p_{0} & +z_{1} p_{1} & +\cdots & +z_{n} p_{n} & =\mu_{1}, \\
& z_{0}^{2} p_{0} & +z_{1}^{2} p_{1} & +\cdots & +z_{n}^{2} p_{n} & =\mu_{2}, \\
& & & & \vdots \\
& & & \\
& & & & \\
z_{0}^{m} p_{0} & +z_{1}^{m} p_{1} & +\cdots & +z_{n}^{m} p_{n} & =\mu_{m}, \\
p_{0}, & p_{1}, & \cdots & p_{n} & \geq 0,
\end{array}
$$

where the unknown variables are $p_{i}, i=0,1, \ldots, n$. The support $I=\left\{z_{0}, z_{1}, \ldots, z_{n}\right\}$, the values of the function $f(z), z \in I$, and the moments $\mu_{k}, k=0,1, \ldots, m$, are given. Since the coefficient matrix of (1.2) is an ill-conditioned Vandermonde matrix, the problem usually cannot be solved by general solution methods. However, under some conditions of the function $f$, Prékopa [27] developed a numerically stable dual method for the solution. This method is based on theorems which give the subscript structures of columns of all dual feasible bases. By the aid of the known dual feasible bases, closed form bounds in terms of the moments can also be given; see Boros and Prékopa [3].

The third useful property is that the optimal solution of the so-called binomial moment problem can give sharp Bonferroni-type bounds and other probability bounds, as well. The $k$ th binomial moment of a random variable $X$ with the support $I \subset \mathbb{N}$ is defined as

$$
E\left[\left(\begin{array}{l}
X \\
k
\end{array}\right)\right]
$$

Let $A_{1}, A_{2}, \ldots, A_{n}$ be arbitrary events. Let the random variable $X$ with the support $\{0,1, \ldots, n\}$ be the number of those events which occur. Then the binomial moments of $X$ equals the following (see, e.g., Prékopa [29, p. 182]):

$$
E\left[\left(\begin{array}{l}
X \\
k
\end{array}\right)\right]=S_{k}=\sum_{0 \leq i_{1}<i_{2}<\cdots<i_{k} \leq n} P\left(A_{i_{1}} \cap A_{i_{2}} \cap \cdots \cap A_{i_{k}}\right),
$$

$k=1,2, \ldots$, and also $E\left[\left(\begin{array}{l}X \\ 0\end{array}\right)\right]=S_{0}=1$. The binomial moment problem has the following form: 


$$
\begin{aligned}
& \min (\max ) \quad f_{0} p_{0} \quad+f_{1} p_{1} \quad+\cdots \quad+f_{n} p_{n} \\
& \text { subject to } \\
& p_{0} \quad+p_{1}+\cdots \quad+p_{n}=S_{0}(=1), \\
& \left(\begin{array}{l}
0 \\
1
\end{array}\right) p_{0}+\left(\begin{array}{l}
1 \\
1
\end{array}\right) p_{1}+\cdots+\left(\begin{array}{l}
n \\
1
\end{array}\right) p_{n}=S_{1}, \\
& \left(\begin{array}{l}
0 \\
2
\end{array}\right) p_{0} \quad+\left(\begin{array}{l}
1 \\
2
\end{array}\right) p_{1} \quad+\cdots+\left(\begin{array}{c}
n \\
2
\end{array}\right) p_{n}=S_{2}, \\
& \left(\begin{array}{c}
0 \\
m
\end{array}\right) p_{0}+\left(\begin{array}{c}
1 \\
m
\end{array}\right) p_{1}+\cdots+\left(\begin{array}{c}
n \\
m
\end{array}\right) p_{n}=S_{m},
\end{aligned}
$$

If we would like to give bounds for the probability of the union or intersection of all the $n$ events, then we have to consider

$$
f(z)=\left\{\begin{array}{l}
0 \text { if } z=0, \\
1 \text { otherwise }
\end{array} \quad \text { or } f(z)=\left\{\begin{array}{l}
1 \text { if } z=s \\
0 \text { otherwise }
\end{array}\right.\right.
$$

respectively. If in problem (1.2) we assume that $\left\{z_{0}, z_{1}, \ldots, z_{n}\right\}=\{0,1, \ldots n\}$, then problems (1.2) and (1.4) can be transformed into each other by simple nonsingular transformations (see Prékopa [25]). This means that the matrices of the equality constraints can be transformed into each other by a nonsingular square matrix and its inverse, respectively. This fact implies that a basis in problem (1.2) is dual feasible if and only if it is dual feasible in (1.4). Hence, the dual method of Prékopa [27] can be applied as well as closed form bounds can be given for the binomial DMP, too. If we consider the first function in (1.5), then sharp Bonferroni-type bounds can be given for the union of events in terms of $S_{0}, S_{1}, \ldots, S_{m}$; see Prékopa [26], [28], [29] and Boros and Prékopa [3].

1.2. The multivariate discrete moment problem. The multivariate discrete moment problem (MDMP) has been introduced by Prékopa [28] and also discussed in papers by Prékopa [30], [32], Mádi-Nagy and Prékopa [21], and Mádi-Nagy [20]. The MDMP can be formulated as follows. Let $\boldsymbol{X}=\left(X_{1}, \ldots, X_{s}\right)$ be a random vector and assume that the support of $X_{j}$ is a known finite set $Z_{j}=\left\{z_{j 0}, \ldots, z_{j n_{j}}\right\}$, consisting of distinct elements, $j=1, \ldots, s$. A certain set of the following moments will be considered.

Definition 1.1. The $\left(\alpha_{1}, \ldots, \alpha_{s}\right)$-order power moment of the random vector $\left(X_{1}, \ldots, X_{s}\right)$ is defined as

$$
\mu_{\alpha_{1} \ldots \alpha_{s}}=E\left[X_{1}^{\alpha_{1}} \cdots X_{s}^{\alpha_{s}}\right]
$$

where $\alpha_{1}, \ldots, \alpha_{s}$ are nonnegative integers. The sum $\alpha_{1}+\cdots+\alpha_{s}$ will be called the total order of the moment.

We use the following notation for the (unknown) distribution of $\boldsymbol{X}$ :

$$
p_{i_{1} \ldots i_{s}}=P\left(X_{1}=z_{1 i_{1}}, \ldots, X_{s}=z_{s i_{s}}\right), \quad 0 \leq i_{j} \leq n_{j}, j=1, \ldots, s .
$$

Then the moments can be written in the form

$$
\mu_{\alpha_{1} \ldots \alpha_{s}}=\sum_{i_{1}=0}^{n_{1}} \cdots \sum_{i_{s}=0}^{n_{s}} z_{1 i_{1}}^{\alpha_{1}} \cdots z_{s i_{s}}^{\alpha_{s}} p_{i_{1} \ldots i_{s}} .
$$

Let $Z=Z_{1} \times \cdots \times Z_{s}$ and $f(\boldsymbol{z}), \boldsymbol{z} \in Z$, be a function. Let

$$
f_{i_{1} \ldots i_{s}}=f\left(z_{1 i_{1}}, \ldots, z_{s i_{s}}\right) .
$$


The (power) MDMP is to give bounds for

$$
E\left[f\left(X_{1}, \ldots, X_{s}\right)\right],
$$

where distribution of $X$ (i.e., (1.6)) is unknown, but known are the moments

$$
\mu_{\alpha_{1} \ldots \alpha_{s}} \text { for }\left(\alpha_{1} \ldots \alpha_{s}\right) \in H
$$

We can formulate the problem by the following LP:

$$
\min (\max ) \sum_{i_{1}=0}^{n_{1}} \cdots \sum_{i_{s}=0}^{n_{s}} f_{i_{1} \ldots i_{s}} p_{i_{1} \ldots i_{s}}
$$

subject to

$$
\begin{aligned}
& \sum_{i_{1}=0}^{n_{1}} \cdots \sum_{i_{s}=0}^{n_{s}} z_{1 i_{1}}^{\alpha_{1}} \cdots z_{s i_{s}}^{\alpha_{s}} p_{i_{1} \ldots i_{s}}=\mu_{\alpha_{1} \ldots \alpha_{s}} \\
& \quad \text { for }\left(\alpha_{1} \ldots \alpha_{s}\right) \in H, \\
& p_{i_{1} \ldots i_{s}} \geq 0 \text { for all } i_{1}, \ldots, i_{s} .
\end{aligned}
$$

In problem (1.7) $p_{i_{1} \ldots i_{s}}, 0 \leq i_{j} \leq n_{j}, j=1, \ldots, s$, are the unknown variables; all other parameters (i.e., the function $f$ and the moments) are given. Regarding the set $H$, in the literature the following are considered. In Prékopa [30], [32]

$$
H=\left\{\left(\alpha_{1}, \ldots, \alpha_{s}\right) \mid 0 \leq \alpha_{j}, \alpha_{j} \text { integer, } \alpha_{1}+\cdots+\alpha_{s} \leq m, j=1, \ldots, s\right\}
$$

where $m$ is a given nonnegative integer, and

$$
H=\left\{\left(\alpha_{1} \ldots \alpha_{s}\right) \mid 0 \leq \alpha_{j} \leq m_{j}, \alpha_{j} \text { integer, } j=1, \ldots, s\right\},
$$

where $m_{j}, j=1, \ldots, s$, are given nonnegative integers. In Mádi-Nagy and Prékopa [21] and Mádi-Nagy [20]

$$
\begin{aligned}
H= & \left\{\left(\alpha_{1}, \ldots, \alpha_{s}\right) \mid 0 \leq \alpha_{j}, \alpha_{j} \text { integer, } \alpha_{1}+\cdots+\alpha_{s} \leq m, j=1, \ldots, s ;\right. \\
& \text { or } \left.\alpha_{j}=0, j=1, \ldots, k-1, k+1, \ldots, s, m \leq \alpha_{k} \leq m_{k}, k=1, \ldots, s\right\}
\end{aligned}
$$

was considered.

The MDMP, beside arising in a natural way, can be applied in several other fields, e.g., bounding expected utilities (Prékopa and Mádi-Nagy [35]), solving generalized $s$-dimensional transportation problems (Hou and Prékopa [16]) and approximating values of multivariate generating functions (Mádi-Nagy and Prékopa [22]). One of the most popular applications is to bound probabilities of Boolean functions of events. These results are based on the binomial MDMP. Let us introduce the notion of crossbinomial moments.

Definition 1.2. The $\left(\alpha_{1}, \ldots, \alpha_{s}\right)$-order cross-binomial moment of the random vector $\left(X_{1}, \ldots, X_{s}\right)$, with the support $Z \subset \mathbb{N}^{s}$, is defined as

$$
S_{\alpha_{1} \ldots \alpha_{s}}=E\left[\left(\begin{array}{c}
X_{1} \\
\alpha_{1}
\end{array}\right) \cdots\left(\begin{array}{c}
X_{s} \\
\alpha_{s}
\end{array}\right)\right]
$$

where $\alpha_{1}, \ldots, \alpha_{s}$ are nonnegative integers. The sum $\alpha_{1}+\cdots+\alpha_{s}$ will be called the total order of the moment.

Assume that we have $n$ arbitrary events. We can subdivide them into $s$ subsequences. Let the $j$ th subsequence be designated as $A_{j 1}, \ldots, A_{j n_{j}}, j=1, \ldots, s$. 
Certainly, $n_{1}+\cdots+n_{s}=n$. Let the random variable $X_{j}$ with the support $Z_{j}=$ $\left\{0,1, \ldots, n_{j}\right\}$ be the number of events that occur in the $j$ th sequence, $j=1, \ldots, s$. In case of event sequences

$$
E\left[\left(\begin{array}{c}
X_{1} \\
\alpha_{1}
\end{array}\right) \cdots\left(\begin{array}{c}
X_{s} \\
\alpha_{s}
\end{array}\right)\right]=\sum_{\substack{1 \leq i_{j 1}<\cdots<i_{j \alpha_{j}} \leq n_{j} \\
j=1, \ldots, s}} P\left[A_{1 i_{11}} \cap \cdots \cap A_{1 i_{1 \alpha_{1}}} \cap \cdots \cap A_{s i_{s 1}} \cap \cdots \cap A_{s i_{s \alpha_{s}}}\right]
$$

in accordance with the notation $S_{\alpha_{1} \ldots \alpha_{s}}$ of Definition 1.2. The binomial MDMP can be formulated by the following LP:

$$
\min (\max ) \sum_{i_{1}=0}^{n_{1}} \cdots \sum_{i_{s}=0}^{n_{s}} f_{i_{1} \ldots i_{s}} p_{i_{1} \ldots i_{s}}
$$

subject to

$$
\begin{aligned}
& \sum_{i_{1}=0}^{n_{1}} \ldots \sum_{i_{s}=0}^{n_{s}}\left(\begin{array}{c}
i_{1} \\
\alpha_{1}
\end{array}\right) \cdots\left(\begin{array}{c}
i_{s} \\
\alpha_{s}
\end{array}\right) p_{i_{1} \ldots i_{s}}=S_{\alpha_{1} \ldots \alpha_{s}} \\
& \text { for }\left(\alpha_{1} \ldots \alpha_{s}\right) \in H, \\
& p_{i_{1} \ldots i_{s}} \geq 0 \text { for all } i_{1}, \ldots, i_{s} .
\end{aligned}
$$

If we would like to give bounds for the probability of the union or intersection of all the $n$ events, then we have to consider

$$
f\left(z_{1}, \ldots, z_{s}\right)=\left\{\begin{array}{l}
0 \text { if }\left(z_{1}, \ldots, z_{s}\right)=(0, \ldots, 0), \\
1 \text { otherwise }
\end{array}\right.
$$

or

$$
f\left(z_{1}, \ldots, z_{s}\right)=\left\{\begin{array}{l}
1 \text { if }\left(z_{1}, \ldots, z_{s}\right)=\left(n_{1}, \ldots, n_{j}\right), \\
0 \text { otherwise }
\end{array}\right.
$$

respectively. If we assume in problem (1.7) that $Z_{j}=\left\{0,1, \ldots, n_{j}\right\}, j=1, \ldots, s$, then in the case of the set $H$ (1.10) and (1.8), problems (1.7) and (1.11) can be transformed into each other by simple nonsingular transformations, similar to in the univariate case. Hence, the dual feasible basis columns in the binomial MDMP and in its transformed power MDMP pair are the same. The binomial MDMP gives a useful tool to approximate the unknown probabilities, e.g., in network reliability calculation (Habib and Szántai [11]) as well as in probabilistic constrained stochastic programming models (Prékopa [31], Fábián and Szőke [8]). It can also be a good alternative to the bounding techniques of Bukszár and Prékopa [4] and Bukszár and Szántai [5]. This type of probability bound is also useful in developing variance reduction MonteCarlo simulation algorithms for estimating the exact probability values (Szántai [39], [40]).

Unfortunately, in case of the MDMP we cannot give all the dual feasible structures under any assumption on the function $f$; hence, we cannot generalize the numerically stable dual method of DMP for the multivariate case. However, under some circumstances, we can give some dual feasible bases which can be used as an initial basis in the dual method. This means, on one hand, that we can skip the first phase in the execution of the dual algorithm, which results in shorter running time and better numerical stability. On the other hand, the objective function value corresponding to a dual feasible basic solution yields a bound for the optimum value of problem (1.7). Hence, if we know a large variety of dual feasible bases, then the best bounds corresponding to those bases can give a good approximation to the optimum value 
without any numerical difficulties. In case of (1.9) we know enough dual feasible bases for the approximation, but in the case of (1.10) (and (1.8)) a large number of dual feasible bases are known only in the bivariate case (i.e., $s=2$ ).

The main result of this paper is that we generalize the dual feasible basis structures corresponding to the bivariate case of the set $H$ (1.10), for higher dimensions. We follow a similar way as in the Min and Max Algorithms of Mádi-Nagy and Prékopa [21], but we define a new set of $H$ :

$$
\begin{aligned}
H= & \left\{\left(\alpha_{1}, 0, \ldots, 0, \alpha_{j}, 0, \ldots, 0\right) \mid 0 \leq \alpha_{1}, \alpha_{j}, \alpha_{1}, \alpha_{j} \text { integer, } \alpha_{1}+\alpha_{j} \leq m,\right. \\
& j=2, \ldots, s ;\} \\
& \cup\left\{\left(0, \ldots, 0, \alpha_{j}, 0, \ldots, 0\right) \mid m+1 \leq \alpha_{j} \leq m_{j}, \alpha_{j} \text { integer, } j=1, \ldots, s\right\} .
\end{aligned}
$$

It is easy to see that, in the bivariate case, set (1.14) and set (1.10) are the same. The choice of (1.14) allows us to give a large variety of dual feasible bases of higher dimensional MDMPs under some assumptions on the function $f$. In case of $H$ (1.14) the power and the binomial MDMP can be transformed into each other by simple nonsingular transformations, which means that the given dual feasible bases can be used in the binomial MDMP, too. The advantage of a large set of directly given dual feasible bases is twofold. On one hand, we can give good bounds without numerical difficulties even if regular solvers cannot give any useful results. On the other hand, in case of the binomial MDMP, we can take binomial moments (i.e., sums of probabilities of intersections of events) of many subsets of the events into account. Hence, the dual feasible bases of the binomial MDMP can yield better bounds than the bounding methods based on the information of the individual probabilities of the intersection of events, e.g., the method of Bukszár and Prékopa [4]. These advantages will be illustrated by numerical examples.

Our bounding technique of the MDMP is based on multivariate Lagrange interpolation. In section 3 we give a multivariate Lagrange polynomial with prescribed degrees of the variables that approximates the function values of $f\left(z_{1}, \ldots, z_{s}\right)$, $\left(z_{1}, \ldots, z_{s}\right) \in Z$ from below or above under some assumptions on the function $f$.

This paper is organized as follows. In section 2 we present the connection between the MDMP and the multivariate Lagrange interpolation; in section 3 we prove a theorem on multivariate Lagrange interpolation corresponding to our new MDMP. In section 4 we give several dual feasible bases as well as bounds of the MDMP with the set $\mathrm{H}$ (1.14). In section 5 numerical examples are presented.

2. The MDMP and the multivariate Lagrange interpolation. In case of univariate Lagrange interpolation concerning arbitrary distinct points $z_{0}, \ldots, z_{n} \in \mathbb{R}$ a unique interpolation polynomial of degree $n$ can be given. The multivariate case is much more difficult. On one hand, it is difficult to identify the geometric distribution of the interpolation points for which we can give a (unique) multivariate Lagrange polynomial with prescribed degrees of the variables. On the other hand, it is also difficult to give an appropriate reminder formula.

Regarding the degrees of the Lagrange polynomial we give the following definition.

DEFINITION 2.1. Let $H=\left\{\left(\alpha_{1}, \ldots, \alpha_{s}\right)\right\}$ be a finite set of $s$-tuples of nonnegative integers $\left(\alpha_{1}, \ldots, \alpha_{s}\right)$, and $\boldsymbol{z}=\left(z_{1}, \ldots z_{s}\right) \in \mathbb{R}^{s}$. We say that $p(\boldsymbol{z})$ is an $H$-type polynomial if its variables have the degrees from the set $H$, i.e.,

$$
p(\boldsymbol{z})=\sum_{\left(\alpha_{1}, \ldots, \alpha_{s}\right) \in H} c_{\alpha_{1} \cdots \alpha_{s}} z_{1}^{\alpha_{1}} \cdots z_{s}^{\alpha_{s}},
$$

where all $c_{\alpha_{1} \cdots \alpha_{s}}$ are real. 
On the geometric distributions of the interpolation points we can give the following definition.

Definition 2.2. Let $U=\left\{\boldsymbol{u}_{1}, \ldots, \boldsymbol{u}_{M}\right\}$ be a set of distinct points in $\mathbb{R}^{s}$ and $H=\left\{\left(\alpha_{1}, \ldots, \alpha_{s}\right)\right\}$ a finite set of $s$-tuples of nonnegative integers $\left(\alpha_{1}, \ldots, \alpha_{s}\right)$. We say that the set $U$ admits an $H$-type Lagrange interpolation if for any real function $f(\boldsymbol{z}), \boldsymbol{z} \in U$, there exists an $H$-type polynomial $p(\boldsymbol{z})$, such that

$$
p\left(\boldsymbol{u}_{i}\right)=f\left(\boldsymbol{u}_{i}\right), \quad i=1, \ldots, M .
$$

In the literature of multivariate Lagrange interpolation the notion of poised set of points is usual; see, e.g., Definition 1.1 in Gasca and Sauer [9]. Regarding this, Definition 2.2 is equivalent to the notion that the set of points $U$ is poised in the linear space of $H$-type Lagrange polynomials. However, in this paper our definition is more appropriate because, in contrast with other papers on interpolation, we do not deal explicitly with the linear space of polynomials.

The problem of $H$-type Lagrange interpolation in case of set $H$ (1.8) and (1.9) was discussed in several papers. A good survey on this topic with results can be found in Gasca and Sauer [9]; an earlier historical background is presented in Gasca and Sauer [10]. The case of the set $H$ (1.10) was first investigated in Mádi-Nagy and Prékopa [21]. The results of our case (1.14) will be presented in the following section.

Regarding the connection between the MDMP and the multivariate Lagrange interpolation we should consider the following. Let us use the following notation for the compact matrix form of (1.7), for a given set $H$ :

subject to

$$
\min (\max ) \boldsymbol{f}^{T} \boldsymbol{p}
$$

$$
\begin{aligned}
A \boldsymbol{p} & =\boldsymbol{b} \\
\boldsymbol{p} & \geq \mathbf{0} .
\end{aligned}
$$

DeFinition 2.3. Let $\boldsymbol{b}\left(z_{1}, \ldots, z_{s}\right)$ be defined in a similar way as $\boldsymbol{b}$, but we remove the expectation and replace $z_{j}$ for $X_{j}, j=1, \ldots, s$; i.e., if we consider a component of the vector $\boldsymbol{b}$, which can be written as $\mu_{\alpha_{1} \cdots \alpha_{s}}=E\left[X_{1}^{\alpha_{1}} \cdots X_{s}^{\alpha_{s}}\right]\left(\left(\alpha_{1}, \ldots \alpha_{s}\right) \in H\right)$, then the corresponding component of the vector $\boldsymbol{b}\left(z_{1}, \ldots, z_{s}\right)$ will be $z_{1}^{\alpha_{1}} \cdots z_{s}^{\alpha_{s}}$.

TheOREm 2.1. Let us consider a basis $B$ of problem (2.3). Note that the term "basis" as well as the symbol B mean a matrix and, at the same time, the collection of its column vectors. Let I be the set of subscripts corresponding to the columns of B, i.e.,

$$
I=\left\{\left(i_{1}, \ldots, i_{s}\right) \mid a_{i_{1} \ldots i_{s}} \in B\right\},
$$

where $a_{i_{1} \ldots i_{s}}$ indicates the column of the coefficient matrix $A$ corresponding to the point $\left(z_{1 i_{1}}, \ldots, z_{s i_{s}}\right)$. If we consider the set of distinct points in $\mathbb{R}^{s}$

$$
U=\left\{\left(z_{1 i_{1}}, \ldots, z_{s i_{s}}\right) \mid\left(i_{1}, \ldots, i_{s}\right) \in I\right\},
$$

then

$$
L_{I}\left(z_{1}, \ldots, z_{s}\right)=\boldsymbol{f}_{B}^{T} B^{-1} \boldsymbol{b}\left(z_{1}, \ldots, z_{s}\right)
$$

is the unique $H$-type Lagrange polynomial corresponding to the set $U$.

Proof. The proof of fitting the interpolation points is yielded by substitution. The uniqueness follows from the fact that if the vector $\boldsymbol{c}$ consists of the corresponding 
coefficients of an $H$-type Lagrange polynomial fitting the set $U$, then it should be the solution of

$$
\boldsymbol{c}^{T} B=\boldsymbol{f}_{B}^{T} .
$$

Indeed, the only solution of (2.7) is $\boldsymbol{c}^{T}=\boldsymbol{f}_{B}^{T} B^{-1}$.

TheOREM 2.2. Let $V_{\min }\left(V_{\max }\right)$ designate the minimum (maximum) value in problem (2.3). Further let $B_{1}\left(B_{2}\right)$ designate a dual feasible basis, i.e.,

$$
\begin{gathered}
\boldsymbol{f}_{B_{1}}^{T} B_{1}{ }^{-1} \boldsymbol{a}_{i_{1} \cdots i_{s}} \leq f_{i_{1} \cdots i_{s}} \text { for all }\left(z_{i_{1}}, \ldots, z_{i_{s}}\right) \in Z, \\
\left(\boldsymbol{f}_{B_{2}}^{T} B_{2}{ }^{-1} \boldsymbol{a}_{i_{1} \cdots i_{s}} \geq f_{i_{1} \cdots i_{s}} \text { for all }\left(z_{i_{1}}, \ldots, z_{i_{s}}\right) \in Z\right),
\end{gathered}
$$

for the minimization (maximization) problem. Relation (2.8) is called the condition of optimality of the minimization (maximization) problem (2.3). Then

$$
\boldsymbol{f}_{B_{1}}^{T} \boldsymbol{p}_{B_{1}} \leq V_{\min } \leq E\left[f\left(X_{1}, \ldots, X_{s}\right)\right] \leq V_{\max } \leq \boldsymbol{f}_{B_{2}}^{T} \boldsymbol{p}_{B_{2}} .
$$

If $B_{1}\left(B_{2}\right)$ is an optimal basis in the minimization (maximization) problem, then the first (last) inequality holds with equality sign. We say that $V_{\min }$ and $V_{\max }$ are the sharp lower and upper bounds, respectively, for the expectation of $f\left(X_{1}, \ldots, X_{s}\right)$.

Proof. The theorem follows from the basic results of linear programming theory.

By the aid of dual feasible bases we can give a special multivariate Lagrange interpolation that approximates the function values of $f\left(z_{1}, \ldots, z_{s}\right)$ from below (above) in case of the min $(\max )$ problem of (2.3).

THEOREM 2.3. If the basis $B$ is dual feasible in the minimization (maximization) problem and the subscript set $I$ is defined as in (2.4), then

$$
\begin{aligned}
& f\left(z_{1}, \ldots, z_{s}\right) \geq L_{I}\left(z_{1}, \ldots, z_{s}\right) \text { for all }\left(z_{1}, \ldots, z_{s}\right) \in Z, \\
& \left(f\left(z_{1}, \ldots, z_{s}\right) \leq L_{I}\left(z_{1}, \ldots, z_{s}\right) \text { for all }\left(z_{1}, \ldots, z_{s}\right) \in Z\right),
\end{aligned}
$$

where equality holds in case of $\left(z_{1}, \ldots, z_{s}\right) \in U$ of $(2.5)$. Regarding $E\left[f\left(X_{1}, \ldots, X_{s}\right)\right]$ we have the following bound:

$$
\begin{aligned}
& E\left[f\left(X_{1}, \ldots, X_{s}\right)\right] \geq E\left[L_{I}\left(X_{1}, \ldots, X_{s}\right)\right], \\
& \left(E\left[f\left(X_{1}, \ldots, X_{s}\right)\right] \leq E\left[L_{I}\left(X_{1}, \ldots, X_{s}\right)\right]\right) .
\end{aligned}
$$

If the basis is also primal feasible, then it is optimal and, thus, the obtained bound is sharp.

Proof. The inequality (2.10) follows from (2.6) and (2.8). The bound (2.11) can be obtained if we replace $\left(X_{1}, \ldots, X_{s}\right)$ for $\left(z_{1}, \ldots, z_{s}\right)$ and take the expectations in (2.10).

TheOREM 2.4. Assume that $L_{I}(\boldsymbol{z})$ is an $H$-type Lagrange polynomial corresponding to the points $Z_{I}$ and inequality (2.10) is satisfied. If the columns in the min (max) problem (2.3) corresponding to the interpolation points $Z_{I}$ are linearly independent, then they form a dual feasible basis.

Proof. Let $B_{1}\left(B_{2}\right)$ be the matrix that consists of the columns corresponding to the points of $Z_{I}$. Since $B_{1}\left(B_{2}\right)$ is basis, $(2.10)$ is equivalent to the condition of optimality (2.8). Thus, $B_{1}\left(B_{2}\right)$ is dual feasible in the min $(\max )$ problem $(2.3)$.

In the following section we give a formula of an $H$-type Lagrange polynomial and its remainder, where the set $H$ is the type of (1.14). We fix only the subscript set $I$ of the interpolation points, which means that we can get several sets

$$
Z_{I}=\left\{\left(z_{1 i_{1}}, \ldots, z_{s i_{s}}\right) \mid\left(i_{1}, \ldots, i_{s}\right) \in I\right\}
$$


that admit an $H$-type Lagrange interpolation. Indeed, if we change the order of the elements in the sequence $\left\{z_{j 0}, \ldots, z_{j n_{j}}\right\}$ (or, equivalently, we change the subscripts of the elements within the sets $\left.Z_{j}\right), j=1, \ldots, s$, then the set of interpolation points $Z_{I}$ will be different.

The coefficients of the Lagrange polynomial will be given in terms of the multivariate divided differences of the function $f$. Hence, we give the following definition.

DeFINITION 2.4. Let $f(z), z \in\left\{z_{0}, \ldots, z_{n}\right\}$, be a univariate discrete function, where $z_{0}, \ldots, z_{n}$ are distinct real numbers,

$$
\left[z_{i} ; f\right]:=f\left(z_{i}\right), \text { where } z_{i} \in\left\{z_{0}, \ldots, z_{n}\right\} .
$$

The $k t h$ order (univariate) divided differences $(k \geq 1)$ are defined recursively:

$$
\left[z_{i}, \ldots, z_{i+k} ; f\right]=\frac{\left[z_{i+1}, \ldots, z_{i+k} ; f\right]-\left[z_{i}, \ldots, z_{i+k-1} ; f\right]}{z_{i+k}-z_{i}}, \text { where } z_{i} \in\left\{z_{0}, \ldots, z_{n}\right\} .
$$

Definition 2.5. Let $f(\boldsymbol{z}), \boldsymbol{z} \in Z=Z_{1} \times \cdots \times Z_{s}$, be a multivariate discrete function and take the subset

$$
\begin{aligned}
Z_{I_{1} \ldots I_{s}} & =\left\{z_{1 i}, i \in I_{1}\right\} \times \cdots \times\left\{z_{s i}, i \in I_{s}\right\} \\
& =Z_{1 I_{1}} \times \cdots \times Z_{s I_{s}}
\end{aligned}
$$

where $\left|I_{j}\right|=k_{j}+1, j=1, \ldots, s$. Then we can define the $\left(k_{1}, \ldots, k_{s}\right)$-order (multivariate) divided difference of $f$ on the set (2.13) in an iterative way. First we take the $k_{1}$ th divided difference with respect to the first variable, then the $k_{2}$ th divided difference with respect to the second variable, etc. These operations can be executed in any order even in a mixed manner, the result always being the same. Let

$$
\left[z_{1 i}, i \in I_{1} ; \cdots ; z_{s i}, i \in I_{s} ; f\right]
$$

designate the $\left(k_{1}, \ldots, k_{s}\right)$-order divided difference. The sum $k_{1}+\cdots+k_{s}$ is called the total order of the divided difference.

In order to make the definition easier to understand we present the following example.

Example 2.1.

$$
\begin{gathered}
{\left[z_{10}, z_{11} ; z_{20}, z_{21} ; f\right]=\left[z_{20}, z_{21} ; \frac{f\left(z_{11}, z_{2}\right)-f\left(z_{10}, z_{2}\right)}{z_{11}-z_{10}}\right]} \\
=\frac{\frac{f\left(z_{11}, z_{21}\right)-f\left(z_{10}, z_{21}\right)}{z_{11}-z_{10}}-\frac{f\left(z_{11}, z_{20}\right)-f\left(z_{10}, z_{20}\right)}{z_{11}-z_{10}}}{z_{21}-z_{20}} .
\end{gathered}
$$

3. A theorem on multivariate Lagrange interpolation. Let $f(z), z \in Z=$ $Z_{1} \times \cdots \times Z_{s}$, where $Z_{j}=\left\{z_{j 0}, \ldots, z_{j n_{j}}\right\}$ consists of distinct real values, $j=1, \ldots, s$. In this section we present an $H$-type Lagrange polynomial and its remainder on the interpolation points $Z_{I}=\left\{\left(z_{1 i_{1}}, \ldots, z_{s i_{s}}\right) \mid\left(i_{1}, \ldots, i_{s}\right) \in I\right\}$, where the set $H$ is defined by (1.14) and

$$
I=\left(\bigcup_{j=1}^{s} I_{j}\right) \cup\left(\bigcup_{j=1}^{s} J_{j}\right)
$$


where

$$
I_{1}=\left\{\left(i_{1}, 0, \ldots, 0\right) \mid 0 \leq i_{1} \leq m-1, \text { integers }\right\},
$$

$$
\begin{gathered}
I_{j}=\left\{\left(i_{1}, 0, \ldots, 0, i_{j}, 0, \ldots, 0\right) \mid \begin{array}{c}
\left.0 \leq i_{1}, 1 \leq i_{j} \leq m-1, \text { integers, } i_{1}+i_{j} \leq m\right\} \\
j=2, \ldots, s
\end{array}\right.
\end{gathered}
$$

and

$$
\begin{aligned}
& J_{j}=\left\{\left(0, \ldots, 0, i_{j}, 0, \ldots, 0\right) \mid i_{j} \in K_{j}\right\}, \\
& K_{j}=\left\{k_{j}^{(1)}, \ldots, k_{j}^{\left(\left|K_{j}\right|\right)}\right\} \subset\left\{m, m+1, \ldots, n_{j}\right\}, \\
& \left|K_{j}\right|=m j+1-m, j=1, \ldots, s .
\end{aligned}
$$

In what follows we will use the notations

$$
\begin{aligned}
Z_{j i}= & \left\{z_{j 0}, \ldots, z_{j i}\right\} \\
Z_{j i}^{\prime}= & \left\{z_{j 0}, \ldots, z_{j i}, z_{j}\right\} \\
& i=0, \ldots, n_{j}, j=1, \ldots, s
\end{aligned}
$$

and

$$
\begin{aligned}
K_{j i}= & \left\{k_{j}^{(1)}, \ldots, k_{j}^{(i)}\right\} \\
Z_{j K_{j i}}= & \left\{z_{j k_{j}^{(1)}}, \ldots, z_{j k_{j}^{(i)}}\right\}, \\
& i=1, \ldots,\left|K_{j}\right|, j=1, \ldots, s, \\
Z_{j K_{j}}= & Z_{j K_{j \mid K_{j}} \mid}, j=1, \ldots, s .
\end{aligned}
$$

We assign the Lagrange polynomial, given by its Newton form

$$
\begin{gathered}
L_{I}\left(z_{1}, \ldots, z_{s}\right) \\
=\sum_{i_{1}=0}^{m-1}\left[Z_{1 i_{1}} ; Z_{20} ; \cdots ; Z_{s 0} ; f\right] \prod_{k=0}^{i_{1}-1}\left(z_{1}-z_{1 k}\right) \\
+\sum_{j=2}^{s} \sum_{\substack{i_{1}+i_{j} \leq m \\
1 \leq i_{j} \leq m-1}}\left[Z_{1 i_{1}} ; Z_{20} ; \cdots ; Z_{(j-1) 0} ; Z_{j i_{j}} ; Z_{(j+1) 0} ; Z_{s 0} ; f\right] \\
\times \prod_{k=0}^{i_{1}-1}\left(z_{1}-z_{1 k}\right) \prod_{k=0}^{i_{j}-1}\left(z_{j}-z_{j k}\right) \\
+\sum_{j=1}^{s} \sum_{i=1}^{\left|K_{j}\right|}\left[Z_{10} ; \cdots ; Z_{(j-1) 0} ; Z_{j(m-1)} \cup Z_{j K_{j i}} ; Z_{(j+1) 0} ; \cdots ; Z_{s 0} ; f\right] \\
\times \prod_{k \in\{0, \ldots, m-1\} \cup K_{j(i-1)}}\left(z_{j}-z_{j k}\right), \\
\text { where, by definition, } \prod_{k=0}^{i_{j}-1}\left(z_{j}-z_{j k}\right)=1, \text { for } i_{j}=0, \text { and } K_{j 0}=\emptyset .
\end{gathered}
$$

In (3.4) the function $f$ is not necessarily restricted to the set $Z$ as its domain of definition; it may be defined on any subset of $\mathbb{R}^{s}$ that contains $Z$.

Next, we define the residual function:

$$
R_{I}\left(z_{1}, \ldots, z_{s}\right)=R_{1 I}\left(z_{1}, \ldots, z_{s}\right)+R_{2 I}\left(z_{1}, \ldots, z_{s}\right)+R_{3 I}\left(z_{1}, \ldots, z_{s}\right),
$$


where

$$
\begin{gathered}
R_{1 I}\left(z_{1}, \ldots, z_{s}\right) \\
=\sum_{j=1}^{s}\left[z_{10} ; \cdots ; z_{(j-1) 0} ; Z_{j(m-1)} \cup Z_{j K_{j}} \cup\left\{z_{j}\right\} ; z_{(j+1) 0} ; \cdots ; z_{s 0} ; f\right] \\
\times \prod_{k \in\{0, \ldots, m-1\} \cup K_{j}}\left(z_{j}-z_{j k}\right)
\end{gathered}
$$

and

$$
\begin{aligned}
& R_{2 I}\left(z_{1}, \ldots, z_{s}\right) \\
& =\sum_{j=2}^{s}\left(\sum_{\substack{i_{1}+i_{j}=m \\
0 \leq i_{1}, i_{j} \leq m-1}}\left[Z_{1 i_{1}}^{\prime} ; Z_{20} ; \cdots ; Z_{(j-1) 0} ; Z_{j i_{j}} ; Z_{(j+1) 0} ; \cdots ; Z_{s 0} ; f\right]\right. \\
& \times \prod_{l=0}^{i_{1}}\left(z_{1}-z_{1 l}\right) \prod_{k=0}^{i_{j}-1}\left(z_{j}-z_{j k}\right) \\
& +\left[Z_{10}^{\prime} ; Z_{20} ; \cdots ; Z_{(j-1) 0} ; Z_{j(m-1)}^{\prime} ; Z_{(j+1) 0} ; \cdots ; Z_{s 0} ; f\right] \\
& \left.\times\left(z_{1}-z_{10}\right) \prod_{k=0}^{m-1}\left(z_{j}-z_{j k}\right)\right)
\end{aligned}
$$

and

$$
\begin{gathered}
R_{3 I}\left(z_{1}, \ldots, z_{s}\right) \\
(3.8)=\sum_{h=2}^{s-1} \sum_{j=h+1}^{s}\left[z_{1} ; \cdots ; z_{h-1} ; Z_{h 0}^{\prime} ; Z_{(h+1) 0} ; \cdots ; Z_{(j-1) 0} ; Z_{j 0}^{\prime} ; Z_{(j+1) 0} ; \cdots ; Z_{s 0} ; f\right] \\
\times\left(z_{h}-z_{h 0}\right)\left(z_{j}-z_{j 0}\right) .
\end{gathered}
$$

We prove the following theorem.

TheOrem 3.1. Consider the H-type Lagrange polynomial (3.4), corresponding to the points $Z_{I}$. For any $\boldsymbol{z}=\left(z_{1}, \ldots, z_{s}\right)$ for which the function $f$ is defined, we have the equality

$$
L_{I}\left(z_{1}, \ldots, z_{s}\right)+R_{I}\left(z_{1}, \ldots, z_{s}\right)=f\left(z_{1}, \ldots, z_{s}\right) .
$$

Proof. For the sake of simplicity we assume that $m_{j} \leq n_{j}, j=1, \ldots, s$. The proof of the general case needs only slight modification. Now we consider the following lemma.

Lemma 3.2. We have the equality

$$
\begin{aligned}
& L_{I}\left(z_{1}, \ldots, z_{s}\right)+R_{1 I}\left(z_{1}, \ldots, z_{s}\right) \\
& =\sum_{i_{1}=0}^{m-1}\left[Z_{1 i_{1}} ; Z_{20} ; \cdots ; Z_{s 0} ; f\right] \prod_{k=0}^{i_{1}-1}\left(z_{1}-z_{1 k}\right) \\
& +\sum_{j=2}^{s} \sum_{\substack{i_{1}+i_{j} \leq m \\
1 \leq i_{j} \leq m-1}}\left[Z_{1 i_{1}} ; Z_{20} ; \cdots ; Z_{(j-1) 0} ; Z_{j i_{j}} ; Z_{(j+1) 0} ; Z_{s 0} ; f\right] \\
& \times \prod_{\substack{i_{1} \\
i_{1}-1}}^{i_{j}-1}\left(z_{1}-z_{1 l}\right) \prod_{k=0}^{s}\left(z_{j}-z_{j k}\right) \\
& +\sum_{j=1}^{s}\left[Z_{10} ; \cdots ; Z_{(j-1) 0} ; Z_{j(m-1)}^{\prime} ; Z_{(j+1) 0} ; \cdots ; Z_{s 0} ; f\right] \prod_{k=0}^{m-1}\left(z_{j}-z_{j k}\right) .
\end{aligned}
$$


Proof of Lemma 3.2. The proof is the same as that of Lemma 3.2 in Mádi-Nagy and Prékopa [21].

LEMMA 3.3.

$$
\begin{aligned}
& L_{I}\left(z_{1}, \ldots, z_{s}\right)+R_{1 I}\left(z_{1}, \ldots, z_{s}\right)+R_{2 I}\left(z_{1}, \ldots, z_{s}\right) \\
& =\left[z_{1} ; Z_{20} ; \cdots ; Z_{s 0} ; f\right] \\
& +\sum_{j=2}^{s}\left[z_{1} ; Z_{20} ; \cdots ; Z_{(j-1) 0} ; Z_{j 0}^{\prime} ; Z_{(j+1) 0} ; \cdots ; Z_{s 0} ; f\right]\left(z_{j}-z_{j 0}\right) .
\end{aligned}
$$

Proof of Lemma 3.3. Let us look at the terms of (3.7) and (3.11) as univariate functions of $z_{1}$. Adding up the first term of (3.10) and the case of $j=1$ in the third term of (3.10) we get the first term of (3.11); i.e.,

$$
\begin{gathered}
\sum_{i_{1}=0}^{m-1}\left[Z_{1 i_{1}} ; Z_{20} ; \cdots ; Z_{s 0} ; f\right] \prod_{k=0}^{i_{1}-1}\left(z_{1}-z_{1 k}\right)+\left[Z_{1(m-1)}^{\prime} ; Z_{20} ; \cdots ; Z_{s 0} ; f\right] \prod_{k=0}^{m-1}\left(z_{1}-z_{1 k}\right) \\
=\left[z_{1} ; Z_{20} ; \cdots ; Z_{s 0} ; f\right] .
\end{gathered}
$$

Let us consider the terms of the second sum of (3.10) for a given value of $j$ and $i_{j}$. Adding them to the terms of the first part of the sum of (3.7) with the same value of $j$ and $i_{j}$ we have

$$
\begin{gathered}
\sum_{i_{1} \leq m-i_{j}}\left[Z_{1 i_{1}} ; Z_{20} ; \cdots ; Z_{(j-1) 0} ; Z_{j i_{j}} ; Z_{(j+1) 0} ; Z_{s 0} ; f\right] \prod_{k=0}^{i_{j}-1}\left(z_{j}-z_{j k}\right) \prod_{l=0}^{i_{1}-1}\left(z_{1}-z_{1 l}\right) \\
+\left[Z_{1\left(m-i_{j}\right)}^{\prime} ; Z_{20} ; \cdots ; Z_{(j-1) 0} ; Z_{j i_{j}} ; Z_{(j+1) 0} ; \cdots ; Z_{s 0} ; f\right] \prod_{k=0}^{i_{j}-1}\left(z_{j}-z_{j k}\right) \prod_{l=0}^{m-i_{j}}\left(z_{1}-z_{1 l}\right) \\
\quad=\left[z_{1} ; Z_{20} ; \cdots ; Z_{(j-1) 0} ; Z_{j i_{j}} ; Z_{(j+1) 0} ; \cdots ; Z_{s 0} ; f\right] \prod_{k=0}^{i_{j}-1}\left(z_{j}-z_{j k}\right) .
\end{gathered}
$$

Adding up all terms of (3.10) and (3.7) corresponding to a given $2 \leq j \leq s$, by the use of the result (3.13) we have

$$
\begin{gathered}
\sum_{i_{j}=1}^{m-1}\left[z_{1} ; Z_{20} ; \cdots ; Z_{(j-1) 0} ; Z_{j i_{j}} ; Z_{(j+1) 0} ; \cdots ; Z_{s 0} ; f\right] \prod_{k=0}^{i_{j}-1}\left(z_{j}-z_{j k}\right) \\
\quad+\left[Z_{10} ; \cdots ; Z_{(j-1) 0} ; Z_{j(m-1)}^{\prime} ; Z_{(j+1) 0} ; \cdots ; Z_{s 0} ; f\right] \prod_{k=0}^{m-1}\left(z_{j}-z_{j k}\right) \\
+\left[Z_{10}^{\prime} ; Z_{20} ; \cdots ; Z_{(j-1) 0} ; Z_{j(m-1)}^{\prime} ; Z_{(j+1) 0} ; \cdots ; Z_{s 0} ; f\right]\left(z_{1}-z_{10}\right) \prod_{k=0}^{m-1}\left(z_{j}-z_{j k}\right)
\end{gathered}
$$




$$
\begin{aligned}
& =\sum_{i_{j}=1}^{m-1}\left[z_{1} ; Z_{20} ; \cdots ; Z_{(j-1) 0} ; Z_{j i_{j}} ; Z_{(j+1) 0} ; \cdots ; Z_{s 0} ; f\right] \prod_{k=0}^{i_{j}-1}\left(z_{j}-z_{j k}\right) \\
& +\left[z_{1} ; Z_{20} ; \cdots ; Z_{(j-1) 0} ; Z_{j(m-1)}^{\prime} ; Z_{(j+1) 0} ; \cdots ; Z_{s 0} ; f\right] \prod_{k=0}^{m-1}\left(z_{j}-z_{j k}\right) .
\end{aligned}
$$

Considering (3.14) as a function of $z_{j}$ we get that this sum equals

$$
\left[z_{1} ; Z_{20} ; \cdots ; Z_{(j-1) 0} ; Z_{j 0}^{\prime} ; Z_{(j+1) 0} ; \cdots ; Z_{s 0} ; f\right]\left(z_{j}-z_{j 0}\right) \text {. }
$$

Adding up (3.12) and the terms (3.15) for $j=2, \ldots, s$ we get (3.11). Thus, the lemma is proven.

Proof of Theorem 3.1. We prove that

$$
\begin{gathered}
\left(L_{I}\left(z_{1}, \ldots, z_{s}\right)+R_{1 I}\left(z_{1}, \ldots, z_{s}\right)+R_{2 I}\left(z_{1}, \ldots, z_{s}\right)\right)+R_{3 I}\left(z_{1}, \ldots, z_{s}\right) \\
=f\left(z_{1}, \ldots, z_{s}\right)
\end{gathered}
$$

where the brackets emphasize that we will use (3.11). Let us choose the $h=2$ case in the sum of (3.8) and add it to (3.11). Considering them as univariate functions of $z_{2}$ the result is

$$
\begin{gathered}
\sum_{j=3}^{s}\left[z_{1} ; Z_{20}^{\prime} ; Z_{30} ; \cdots ; Z_{(j-1) 0} ; Z_{j 0}^{\prime} ; Z_{(j+1) 0} ; \cdots ; Z_{s 0} ; f\right]\left(z_{2}-z_{20}\right)\left(z_{j}-z_{j 0}\right) \\
+\left[z_{1} ; Z_{20} ; \cdots ; Z_{s 0} ; f\right]+\sum_{j=2}^{s}\left[z_{1} ; Z_{20} ; \cdots ; Z_{(j-1) 0} ; Z_{j 0}^{\prime} ; Z_{(j+1) 0} ; \cdots ; Z_{s 0} ; f\right]\left(z_{j}-z_{j 0}\right) \\
=\left(\left[z_{1} ; Z_{20} ; \cdots ; Z_{s 0} ; f\right]+\left[z_{1} ; Z_{20}^{\prime} ; Z_{30} ; \cdots ; Z_{s 0} ; f\right]\left(z_{2}-z_{20}\right)\right) \\
+\left(\sum_{j=3}^{s}\left[z_{1} ; Z_{20} ; \cdots ; Z_{(j-1) 0} ; Z_{j 0}^{\prime} ; Z_{(j+1) 0} ; \cdots ; Z_{s 0} ; f\right]\left(z_{j}-z_{j 0}\right)\right. \\
\left.+\sum_{j=3}^{s}\left[z_{1} ; Z_{20}^{\prime} ; Z_{30} ; \cdots ; Z_{(j-1) 0} ; Z_{j 0}^{\prime} ; Z_{(j+1) 0} ; \cdots ; Z_{s 0} ; f\right]\left(z_{2}-z_{20}\right)\left(z_{j}-z_{j 0}\right)\right) \\
=\sum_{j=3}^{s}\left[z_{1} ; z_{2} ; Z_{30} ; \cdots ; Z_{(j-1) 0} ; Z_{j 0}^{\prime} ; Z_{(j+1) 0} ; \cdots ; Z_{s 0} ; f\right]\left(z_{j}-z_{j 0}\right) .
\end{gathered}
$$

Adding the $h=3$ case in the sum of (3.8) to (3.16) we get a similar formula with $z_{3}$ in the places of the third variable. Finally, after choosing all the cases $h=2,3, \ldots, s-1$ in (3.8) and adding them in this order to (3.16) we have

$$
\left[z_{1} ; z_{2} ; \cdots, z_{s-1} ; Z_{s 0} ; f\right]+\sum_{j=s}^{s}\left[z_{1} ; z_{2} ; \cdots ; z_{s-1} ; Z_{s 0}^{\prime} ; f\right]\left(z_{j}-z_{j 0}\right) .
$$


Considering this as the function of $z_{s}$, the result is

$$
\left[z_{1} ; z_{2} ; \cdots, z_{s-1} ; z_{s} ; f\right]=f\left(z_{1}, \ldots, z_{s}\right) .
$$

Thus, the theorem is proven.

THEOREM 3.4. The $H$-type Lagrange polynomial (3.4) of the function $f$ corresponding to the points $Z_{I}$ is unique (for a given set $Z$ ). Furthermore, the set of columns $B$ of $A$ in problem (2.3), corresponding to the subscript set I, forms a basis of $A$.

Proof. The formula (3.4) gives a Lagrange polynomial on $Z_{I}$ for any function $f$ on the set $Z$. This means that we can obtain the coefficients of the $H$-type Lagrange polynomial in case of any function $f(\boldsymbol{z}), \boldsymbol{z} \in Z$. Let the matrix $B$ consist of the columns corresponding to the points of $Z_{I}$. The coefficient vector $\boldsymbol{c}_{f}$ of the Lagrange polynomial corresponding to the function $f$ is the solution of the equation

$$
\boldsymbol{c}_{f}^{T} B=\boldsymbol{f}_{B}^{T} .
$$

Since we have Lagrange interpolation for any $f,(3.17)$ has a solution $\boldsymbol{c}_{f}$ in case of any vector $\boldsymbol{f}_{B}$. From this follows that the square matrix $B$ is nonsingular, hence the columns corresponding to the points of $Z_{I}$ form a basis. Thus, the second assertion is proven. Then the uniqueness is the corollary of Theorem 2.1.

4. Bounds for the MDMP with the set $\boldsymbol{H}$ (1.14). The problem in the title of this section is the following LP:

$$
\min (\max ) \sum_{i_{1}=0}^{n_{1}} \ldots \sum_{i_{s}=0}^{n_{s}} f_{i_{1} \ldots i_{s}} p_{i_{1} \ldots i_{s}}
$$

subject to

$$
\begin{aligned}
& \sum_{i_{1}=0}^{n_{1}} \cdots \sum_{i_{s}=0}^{n_{s}} z_{1 i_{1}}^{\alpha_{1}} z_{j i_{j}}^{\alpha_{j}} p_{i_{1} \ldots i_{s}}=\mu_{\alpha_{1} 0 \ldots 0 \alpha_{j} 0 \ldots 0} \\
& \quad \text { for } \alpha_{1}, \alpha_{j} \geq 0, \alpha_{1}+\alpha_{j} \leq m, j=2, \ldots, s \\
& \sum_{n_{1}}^{n_{1}} \cdots \sum_{i_{s}=0}^{n_{s}} z_{j i_{j}}^{\alpha_{j}} p_{i_{1} \ldots i_{s}}=\mu_{0 \ldots 0 \alpha_{j} 0 \ldots 0} \\
& \quad \text { for } m+1 \leq \alpha_{j} \leq m_{j}, j=1, \ldots, s \\
& p_{i_{1} \ldots i_{s}} \geq 0 \text { for all } i_{1}, \ldots, i_{s} .
\end{aligned}
$$

In the following theorems let us designate the coefficient matrix of (4.1) by $A$, the right-hand side vector by $\boldsymbol{b}$, and the coefficient vector of the objective function by $\boldsymbol{f}$, in agreement with $(2.3)$.

We present several dual feasible bases for the min problem of (4.1), which give lower bounds for the objective function. As at the numerical examples we shall see, the best bounds, among the given ones, are usually close to the values of the sharp bounds. This means that we can give good bounds without using LP solvers, which work numerically unstably because of the Vandermonde systems in the coefficient matrix.

First of all, we should introduce some assumptions. They are as follows.

Assumption 1 . The function $f(\boldsymbol{z}), \boldsymbol{z} \in Z$,

(a) has nonnegative univariate divided differences of order $m_{j}+1$ regarding $z_{j}$, $j=1, \ldots, s$,

(b) has nonnegative bivariate divided differences of order $m+1$,

(c) has nonnegative mixed second order divided differences. 
If $f(\boldsymbol{z}), \boldsymbol{z} \in Z$, is derived from a function $\bar{f}(\boldsymbol{z})$ defined in $\bar{Z}=\left[z_{10}, z_{1 n_{1}}\right] \times$ $\cdots \times\left[z_{s 0}, z_{s n_{s}}\right]$ by taking $f(\boldsymbol{z})=\bar{f}(\boldsymbol{z}), \boldsymbol{z} \in Z$, and $\bar{f}(\boldsymbol{z})$ has continuous, nonnegative derivatives of order $\left(k_{1}, \ldots, k_{s}\right)$ in the interior of $\bar{Z}$, then all divided differences of $f(\boldsymbol{z}), \boldsymbol{z} \in Z$, of order $\left(k_{1}, \ldots, k_{s}\right)$ are nonnegative. For further results in this respect, see Popoviciu [24]. This fact helps one find out whether a function satisfies Assumption 4. If in the assumption we require nonpositivity, then we shall see that the following bases will be dual feasible for the max problem and will give upper bounds for (4.1).

We introduce four different structures for $K_{j}$, defined in (3.4), as follows:

$$
\begin{array}{ccc} 
& \left|K_{j}\right| \text { even } & \left|K_{j}\right| \text { odd } \\
\min & u^{(j)}, u^{(j)}+1, \ldots, v^{(j)}, v^{(j)}+1 & m, u^{(j)}, u^{(j)}+1, \ldots, v^{(j)}, v^{(j)}+1 \\
\max & m, u^{(j)}, u^{(j)}+1, \ldots, v^{(j)}, v^{(j)}+1, n_{j} & u^{(j)}, u^{(j)}+1, \ldots, v^{(j)}, v^{(j)}+1, n_{j}
\end{array}
$$

i.e., $K_{j}$ (consists of distinct elements of the subset of $\left\{m, \ldots, n_{j}\right\}$ ) is a set of pairs of consecutive elements completed by $m$ and $n_{j}$ depending on its parity and its (min or $\max )$ type.

As regards the ordering of the elements in the sets $Z_{1}, \ldots, Z_{s}$, we mention separately in each theorem of this section what our assumption is about.

Theorem 4.1. Let $z_{j 0}<z_{j 1}<\cdots<z_{j n_{j}}, j=1, \ldots, s$. Suppose that $K_{j}$ follows the min structure of $(4.2), j=1, \ldots, s$.

Under Assumption $4, L_{I}\left(z_{1}, \ldots, z_{s}\right)$, defined by (3.4), is a unique $H$-type Lagrange polynomial on $Z_{I}$ and satisfies the relations

$$
f\left(z_{1}, \ldots, z_{s}\right) \geq L_{I}\left(z_{1}, \ldots, z_{s}\right),\left(z_{1}, \ldots, z_{s}\right) \in Z .
$$

The set of columns $B$ of $A$ in problem (4.1), with the subscript set $I$, is a dual feasible basis in the minimization problem (4.1), and

$$
E\left[f\left(X_{1}, \ldots, X_{s}\right)\right] \geq E\left[L_{I}\left(X_{1}, \ldots, X_{s}\right)\right] .
$$

If $B$ is also a primal feasible basis in problem (4.1), then the inequality (4.4) is sharp.

Proof. We have only to prove (4.3). The proof of the other parts of the theorem is straightforward from Theorems 3.4, 2.4, and 2.3.

In order to prove (4.3) it is sufficient to show that

$$
R_{I}\left(z_{1}, \ldots, z_{s}\right) \geq 0 \text { for all }\left(z_{1}, \ldots, z_{s}\right) \in Z \text {. }
$$

In fact, we show that all terms in the sum $R_{I}\left(z_{1}, \ldots, z_{s}\right)$ are nonnegative. Considering any term in $R_{1 I}\left(z_{1}, \ldots, z_{s}\right)$ the first factor of the product is a divided difference, which is nonnegative because of Assumption 4, while the last part is

$$
\prod_{k \in\{0, \ldots, m-1\} \cup K_{j}}\left(z_{j}-z_{j k}\right)>0 \text { for } z_{j} \notin\left\{z_{j 0}, \ldots, z_{j(m-1)}\right\} \cup Z_{j K_{j}}
$$

because there are even numbers of negative factors, due to the special structure of $K_{j}$. If $z_{j} \in\left\{z_{j 0}, \ldots, z_{j(m-1)}\right\} \cup Z_{j K_{j}}$, the above product is 0 . This means that any term of $R_{1 I}\left(z_{1}, \ldots, z_{s}\right)$ is nonnegative. Considering a term of the sum $R_{2 I}\left(z_{1}, \ldots, z_{s}\right)$ or $R_{3 I}\left(z_{1}, \ldots, z_{s}\right)$ the first factor of the product is a divided difference, which is nonnegative because of Assumption 4. In the other part of the product at least one of the factors is zero or all factors are positive because of the ordering of $Z_{j}$ 's. 


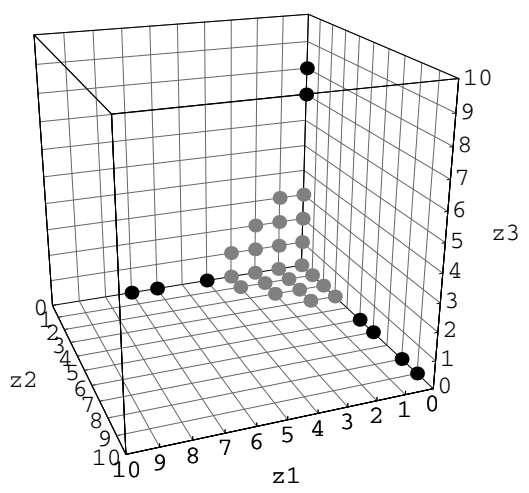

(a)

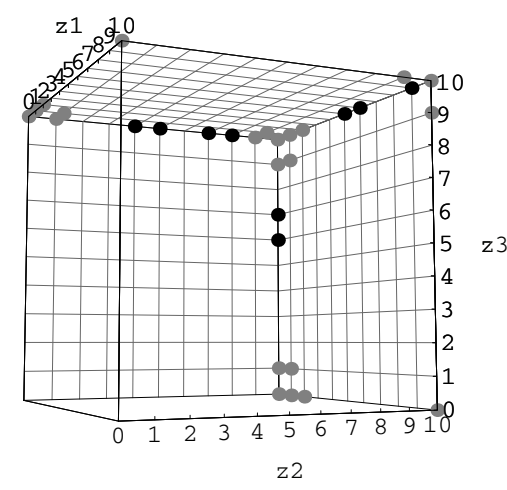

(b)

FIG. 1. Assume $Z_{j}=\{0, \ldots, 10\}, j=1,2,3, m=4, m_{1}=7, m_{2}=8, m_{3}=6$. (a): Dual feasible basis corresponding to Theorem 4.1 , where $K_{1}=\{4,6,7\}, K_{2}=\{5,6,8,9\}, K_{3}=\{7,8\}$. (b): Dual feasible basis of the Min Algorithm, where $\left(z_{10}, z_{11}, z_{12}, z_{13}\right)=(0,1,10,2),\left(z_{j 0}, z_{j 1}, z_{j 2}, z_{j 3}\right)=$ $(10,0,9,1), j=2,3, K_{1}=\{6,7,10\} \quad\left(Z_{1 K_{1}}=\{5,6,9\}\right), K_{2}=\{6,7,9,10\} \quad\left(Z_{2 K_{2}}=\{4,5,7,8\}\right)$, $K_{3}=\{8,9\}\left(Z_{3 K_{3}}=\{6,7\}\right)$. Elements of $\bigcup_{j=1}^{s} I_{j}$ are colored by gray while elements of $\bigcup_{j=1}^{s} J_{j}$ 's are black.

This means that all factors of the product are nonnegative and hence the term is nonnegative as well. All terms of $R_{I}\left(z_{1}, \ldots, z_{s}\right)$ are nonnegative, hence the sum of them, i.e., $R_{I}\left(z_{1}, \ldots, z_{s}\right)$, is also nonnegative on $Z$. Thus, the theorem is proven.

The basis of Theorem 4.1 is illustrated in Figure 1(a).

In the following algorithm we will consider more orders of the elements of the set $Z_{j}$ in the sequence $\left(z_{j 0}, \ldots, z_{j n_{j}}\right), j=1, \ldots, s$, for which all terms of $R_{I}\left(z_{1}, \ldots, z_{s}\right)$ are nonnegative. Note that transposing the elements of the sequence $\left(z_{j 0}, \ldots, z_{j n_{j}}\right)$ is equivalent to exchanging subscripts of the elements; however, the proof of the validity of the algorithm is based on the orders of the elements. At each order of the elements, the set of interpolation points $Z_{I}$ as well as the corresponding dual feasible basis are different, which means that we can give more lower bounds on (4.1) by the objective function value of the dual feasible basis corresponding to $Z_{I}$.

Min Algorithm.

At first we assume, without loss of generality, that $Z_{j}=\left\{0,1, \ldots, n_{j}\right\}, j=$ $1, \ldots, s$.

Step 0. Let

$$
z_{20}=z_{30}=\cdots=z_{s 0}=0
$$

or

$$
z_{20}=n_{2}, z_{30}=n_{3}, \ldots, z_{s 0}=n_{s} .
$$

If (4.7) holds, then let $0 \leq q_{1} \leq m$ be an even number, else (if (4.8) holds) let $0 \leq q_{1} \leq$ $m$ be an odd number. $L:=\left(0,1, \ldots,(m-1)-q_{1}\right), U:=\left(n_{1}, n_{1}-1, \ldots, n_{1}-\left(q_{1}-1\right)\right)$, $V^{0}:=\{$ arbitrary merger of the sequences $L, U\}=\left(v^{0}, v^{1}, \ldots, v^{m-1}\right)$.

$$
\left(z_{10}, \ldots, z_{1(m-1)}\right):=V^{0} .
$$

Let $j=2$. Goto Step 1 . 
Step 1. Initialize $t=0$. If (4.7) holds, then let $l_{0}=1$ and $u_{0}=n_{j}$, else let $l_{0}=0$ and $u_{0}=n_{j}-1$. Goto Step 2 .

Step 2. Let $V^{t}=\left\{v^{0}, v^{1}, \ldots, v^{m-1-t}\right\}, H^{t}=\left\{h^{1}, \ldots, h^{t}\right\}$. If $v^{m-1-t} \in L$, then let $h^{t+1}=l^{t}, l^{t+1}=l^{t}+1, u^{t+1}=u^{t}$, and if $v^{m-1-t} \in U$, then let $h^{t+1}=u^{t}$, $u^{t+1}=u^{t}-1, l^{t+1}=l^{t}$. Set $t \leftarrow t+1$. If $t=m$, then goto Step 3, else repeat Step 2 .

Step 3. Let

$$
\left(z_{j 1}, \ldots, z_{j(m-1)}\right)=H^{m-1} .
$$

Set $j \leftarrow j+1$. If $j=s+1$, then goto Step 4, else goto Step 1 .

Step 4 . Let $0,1, \ldots, r_{j}, n_{j}, \ldots, n_{j}-\left(m-r_{j}-2\right)$ be the numbers used to construct $z_{j 0}, z_{j 1}, \ldots, z_{j(m-1)}$. Then let

$$
\left(z_{j m}, z_{j(m+1)}, \ldots, z_{j n_{j}}\right)=\left(r_{j}+1, r_{j}+2, \ldots, n_{j}-\left(m-r_{j}-1\right)\right)
$$

as ordered sets, $j=1, \ldots, s$. Note that these subsets of the sets $Z_{j}, j=1, \ldots, s$, remain intact. If $m-r_{j}-1$ is even, then $K_{j}$ should follow a minimum structure in (4.2), and if $m-r_{j}-1$ is odd, then $K_{j}$ should follow a maximum structure. Stop, we have completed the construction of the dual feasible basis related to the subscript set $I$.

In the general case, where $Z_{j}$ is not necessarily $\left\{0,1, \ldots, n_{j}\right\}, j=1, \ldots, s$, we do the following. First we order the elements in each $Z_{j}$ in increasing order. Then we establish one-to-one correspondences between the elements of $Z_{j}$ and the elements of the ordered set $\left(0,1, \ldots, n_{j}\right)$. After that, we carry out the Min Algorithm to find a dual feasible basis, using the sets $\left\{0,1, \ldots, n_{j}\right\}, j=1, \ldots, s$. Finally, we create the set $Z_{I}$, by the use of the above mentioned one-to-one correspondences.

THEOREM 4.2. Let the elements of the sequence $\left(z_{j 0}, \ldots, z_{j n_{j}}\right), j=1, \ldots, s$, be in one of the orders of the Min Algorithm, and also let $K_{j}$ follow the min (max) structure if $m-r_{j}-1$ is even (odd) in the Min Algorithm, $j=1, \ldots, s$.

Under Assumption 4, $L_{I}\left(z_{1}, \ldots, z_{s}\right)$, defined by (3.4), is a unique $H$-type Lagrange polynomial on $Z_{I}$ and satisfies the relations

$$
f\left(z_{1}, \ldots, z_{s}\right) \geq L_{I}\left(z_{1}, \ldots, z_{s}\right), \quad\left(z_{1}, \ldots, z_{s}\right) \in Z .
$$

The set of columns $B$ of $A$ in problem (4.1), with the subscript set I, is a dual feasible basis in the minimization problem (4.1), and

$$
E\left[f\left(X_{1}, \ldots, X_{s}\right)\right] \geq E\left[L_{I}\left(X_{1}, \ldots, X_{s}\right)\right] .
$$

If $B$ is also a primal feasible basis in problem (4.1), then the inequality (4.11) is sharp.

Proof. We have only to prove that $R_{I}\left(z_{1}, \ldots, z_{s}\right) \geq 0$ for all $\left(z_{1}, \ldots, z_{s}\right) \in Z$ and then we can follow the proof of Theorem 4.1. We can restrict ourselves to the case $Z_{j}=\left\{0,1, \ldots, n_{j}\right\}, j=1, \ldots, s$, because in the following inequalities only the orders of the elements play a role.

First, let us consider $R_{3 I}\left(z_{1}, \ldots, z_{s}\right)$ of (3.8). All divided differences are nonnegative, and the set of the second parts of the products equals

$$
\left(z_{h}-z_{h 0}\right)\left(z_{j}-z_{j 0}\right), \quad 2 \leq h<j \leq s .
$$

These products can be nonnegative if and only if both factors are nonnegative or both factors are nonpositive. Because we have pairs for all $2 \leq h<j \leq s$ this means that 
either all factors $\left(z_{j}-z_{j 0}\right), 2 \leq j \leq s$, are nonnegative, or all of them are nonpositive. The first case is provided by (4.7) while the second by (4.8). Hence, $R_{3 I}(\boldsymbol{z}) \geq 0$, $z \in Z$.

If we consider $R_{2 I}\left(z_{1}, \ldots, z_{s}\right)$ of (3.7), we will see that the factors of products after the nonnegative divided differences are associated with the following arrays:

$$
\begin{array}{ccccccc}
z_{10} & z_{11} & z_{12} & \cdots & z_{1(m-2)} & z_{1(m-1)} & z_{j 0} \\
z_{10} & z_{11} & z_{12} & \cdots & z_{1(m-2)} & z_{j 0} & z_{j 1} \\
& & & \vdots & & & \\
z_{10} & z_{11} & z_{j 0} & \cdots & z_{j(m-4)} & z_{j(m-3)} & z_{j(m-2)} \\
z_{10} & z_{j 0} & z_{j 1} & \cdots & z_{j(m-3)} & z_{j(m-2)} & z_{j(m-1)} \\
\multicolumn{1}{c}{} & & & \\
& \multicolumn{5}{c}{j=2, \ldots, s .}
\end{array}
$$

A sufficient condition for the nonnegativity of all products in (3.7) is that

$$
\left|\left\{i \mid 0 \leq i \leq i_{1}, z_{1 i}>z_{1}\right\}\right|+\left|\left\{i \mid 0 \leq i \leq i_{j}, z_{j i}>z_{j}\right\}\right|=\text { even number }
$$

for every $0 \leq i_{j} \leq m-1$ integers satisfying $i_{1}+i_{j}=m-1$, and for all $\left(z_{1}, z_{j}\right) \in Z_{1} \times Z_{j}$, $j=2, \ldots, s$. The first $m$ elements of the first row in (4.13) are the elements of $V^{0}$; the $m+1$ st element of the same row is $z_{j 0}$. In Step 0 the parity of $q_{1}$ provides that (4.14) is satisfied for the product of (3.7) corresponding to the first row of (4.13). The elements of $V^{t}, z_{j 0}, H^{t}$, in that order, constitute the $t$ th row of tableau (4.13). In Steps 1 and 2 we define the following element $h^{t+1}$ such that (4.14) is still satisfied for the product of (3.7) corresponding to the $t+1$ st row of (4.13). From this follows that $R_{2 I}(\boldsymbol{z}) \geq 0, \boldsymbol{z} \in Z$.

Regarding $R_{1 I}\left(z_{1}, \ldots, z_{s}\right)$ the divided differences are nonnegative in terms of (3.6). Hence, after the assignments (4.9) we have only to choose the subscript sets $K_{j}$ such that

$$
\prod_{k \in\{0, \ldots, m-1\} \cup K_{j}}\left(z_{j}-z_{j k}\right) \geq 0, \quad \boldsymbol{z} \in Z
$$

for $j=1, \ldots, s$. For each $j$ the products

$$
\prod_{k=0}^{m-1}\left(z_{j}-z_{j k}\right),\left(z_{1}, \ldots, z_{s}\right) \in Z
$$

are nonnegative (nonpositive) if $m-r_{j}-1$ is even (odd). (Note that $r_{2}=\cdots=r_{s}$ by construction.) Then the choice of $K_{j}$ (with assignment (4.9)), $j=1, \ldots, s$, provides that $R_{1 I}(\boldsymbol{z}) \geq 0$ for all $\boldsymbol{z} \in Z$.

Thus, $R_{I}(\boldsymbol{z})=R_{1 I}(\boldsymbol{z})+R_{2 I}(\boldsymbol{z})+R_{3 I}(\boldsymbol{z}) \geq 0$ for all $\boldsymbol{z} \in Z$.

The basis of the algorithm is illustrated in Figure 1(b).

The above algorithm allows for the construction of a variety of dual feasible bases. However, we do not have a simple criterion, like in the dual method, to decide which of the bases, that we can obtain by the above Min Algorithm, would improve on the bound (on the value of the objective function). Hence, in order to give the best bound for $E\left[f\left(X_{1}, \ldots, X_{s}\right)\right]$ we have to calculate the objective function values for all dual feasible bases yielded by the Min Algorithm and then choose the highest value from among them. For a given order of $\left(z_{j 0}, \ldots, z_{j s}\right), j=1, \ldots, s$, we can find the 
best sets $K_{j}, j=1, \ldots, s$, independently. The argument of this can be found in Mádi-Nagy [20].

Numerical experiments show that this method produces very good results much faster than the execution of the dual algorithm. Its other main advantage is that it is not sensitive to the numerical difficulties that arise from the bad numerical property of the matrix $A$.

5. Numerical examples. In the following we consider three numerical examples. In the first one we give bounds for the expected value of a three-variate utility function by the aid of the Min Algorithm of section 4 and we also give the sharp bounds calculated by the LP solver CPLEX (www.ilog.com). The method of the Min Algorithm is written in Wolfram's Mathematica (www.wolfram.com). This means that its running times are not really comparable with the running times of the dual method of CPLEX; however, we give these results, too. Because of the numerical instability of the MDMP, CPLEX sometimes cannot give appropriate results (it reports infeasibility; however, the problem is feasible by construction). These cases are indicated by "-" sign in the tables. We can conclude that our Min Algorithm yields useful bounds without numerical difficulties even if the CPLEX cannot give the right answer.

In the last two examples we give bounds for the union of events by the application of the Min Algorithm for the binomial MDMP (1.11). Comparing the results to the cherry tree bounds of Bukszár and Prékopa [4] and the bounds of univariate and bivariate MDMPs, the bounds of our Min Algorithm turn out to be the best.

Example 5.1. In this example we solve a problem similar to Example 4.2 in Prékopa and Mádi-Nagy [35]. Consider the utility function

$$
\begin{gathered}
u\left(z_{1}, z_{2}, z_{3}\right)=\log \left[\left(e^{\alpha_{1} z_{1}+a_{1}}-1\right)\left(e^{\alpha_{2} z_{2}+a_{2}}-1\right)\left(e^{\alpha_{3} z_{3}+a_{3}}-1\right)-1\right], \\
\left(z_{1}, z_{2}, z_{3}\right) \in Z,
\end{gathered}
$$

where $Z$ is specialized as follows:

$$
Z=(0,1,2,3,4,5,6,7,8,9) \times(0,1,2,3,4,5,6,7,8,9) \times(0,1,2,3,4,5,6,7,8,9) .
$$

Let the parameters be $\alpha_{1}=1.75, \alpha_{2}=1.25, \alpha_{3}=0.75, a_{1}=3, a_{2}=2, a_{3}=1$. In the cited paper it was proven that the even (odd) order derivatives of the function are nonpositive (nonnegative). We use our Min Algorithm for the MDMP (1.7) with set $H$ (1.14) to give an upper bound for the expected utility

$$
E\left[u\left(X_{1}, X_{2}, X_{3}\right)\right] .
$$

Since the even order divided differences of the function $-u\left(z_{1}, z_{2}, z_{3}\right)$ on $Z$ are nonnegative, we can apply the Min Algorithm to give a lower bound for $E\left[-u\left(X_{1}, X_{2}, X_{3}\right)\right]$ that yields, indeed, an upper bound for (5.2).

Regarding the moments taken into account they are generated from the distribution of $\left(X_{1}, X_{2}, X_{3}\right)$, defined by

$$
\begin{aligned}
& X_{1}=\min \left(X+Y_{1}, 9\right), \\
& X_{2}=\min \left(X+Y_{2}, 9\right), \\
& X_{3}=\min \left(X+Y_{3}, 9\right),
\end{aligned}
$$


where $X, Y_{1}, Y_{2}, Y_{3}$ have independent Poisson distributions with parameters 1, 2, 2.5, 3, respectively. Note that $X_{1}, X_{2}, X_{3}$ are stochastically dependent.

We calculate the bounds for several values of the parameters $m, m_{1}, m_{2}, m_{3}$ of the set $H$ (1.14). We also give the sharp upper bounds calculated by the dual method of CPLEX (where it is possible). In order to see the closeness of our (not sharp) upper bounds we also calculate the minimum of the objective function value. That gives the possibility to compare the difference between our upper bound and the dual maximum to the gap between the sharp upper and lower bounds. The results are summarized below.

\begin{tabular}{|c|c|c|c|c|c|c|c|c|c|}
\hline$m$ & $m$ & $m_{2}$ & $m_{3}$ & Upper bound & $\mathrm{CPU}$ & Dual max & $\mathrm{CPU}$ & Dual min & $\mathrm{CPU}$ \\
\hline \begin{tabular}{c|}
3 \\
\end{tabular} & 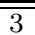 & $\begin{array}{ll}3 \\
\end{array}$ & 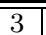 & "18.586715186 & $\overline{00.14}$ & 1818.557668477 & 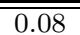 & "18.510511279 & $\overline{0.11}$ \\
\hline 3 & 5 & 5 & 5 & 18.549493662 & 0.41 & 18.546562878 & 0.05 & 18.539798360 & 0.40 \\
\hline 3 & 7 & 7 & 7 & 18.543584031 & 0.69 & 18.543580944 & 0.11 & 18.542882716 & 0.69 \\
\hline 3 & 9 & 9 & 9 & 18.543304428 & 0.36 & - & - & - & - \\
\hline
\end{tabular}

\begin{tabular}{c|c|c|c||c|c||c|c|c|c}
$m$ & $m_{1}$ & $m_{2}$ & $m_{3}$ & Upper bound & CPU & Dual max & CPU & Dual min & CPU \\
\hline \hline 5 & 5 & 5 & 5 & 18.562168728 & 1.50 & 18.546562667 & 0.14 & 18.539911300 & 0.65 \\
5 & 7 & 7 & 7 & 18.543588614 & 2.61 & 18.543580474 & 0.44 & 18.542921460 & 1.16 \\
5 & 9 & 9 & 9 & 18.543304428 & 2.31 & - & - & - & -
\end{tabular}

We can see that our bounds are very close to the sharp bounds except cases $m=m_{1}=\cdots=m_{3}=3$ and $m=m_{1}=\cdots=m_{3}=5$. On the other hand, we can give better approximation taking the marginal moments up to the order 9 into account, despite the fact that CPLEX cannot solve the MDMP.

We also check whether we do not lose too much information using the set $H$ (1.14) instead of the set $H$ (1.10) (set $H$ (1.14) is a subset of $H(1.10)$ ). The sharp bounds for set $H(1.10)$ are summarized below.

\begin{tabular}{c|c|c|c||c|c||c|c}
$m$ & $m_{1}$ & $m_{2}$ & $m_{3}$ & Dual max & CPU & Dual min & CPU \\
\hline \hline 3 & 3 & 3 & 3 & 18.557668337 & 0.28 & 1.8510552138 & 0.12 \\
3 & 5 & 5 & 5 & 18.546562783 & 0.53 & 1.8539798515 & 0.42 \\
3 & 7 & 7 & 7 & 18.543580810 & 1.54 & 1.8542882927 & 0.11 \\
3 & 9 & 9 & 9 & - & - & - & -
\end{tabular}

\begin{tabular}{c|c|c|c||c|c||c|c}
$m$ & $m_{1}$ & $m_{2}$ & $m_{3}$ & Dual max & CPU & Dual min & CPU \\
\hline \hline 5 & 5 & 5 & 5 & 18.546560902 & 2.13 & 1.8539941923 & 1.52 \\
5 & 7 & 7 & 7 & 18.543579438 & 6.62 & 1.8542948216 & 3.69 \\
5 & 9 & 9 & 9 & - & - & - & -
\end{tabular}

We can conclude that these bounds are not significantly better. Hence, we do not lose as much as we can gain on the structure of the set $H$ (1.14), which makes the Min Algorithm applicable for higher dimensions.

Example 5.2. In this example we shall give upper bounds for the probability of the union of events of the following two systems. In both systems there are 12 events $A_{1}, A_{2}, \ldots, A_{12}$, and 16 outcomes, $x_{1}, x_{2}, \ldots x_{16}$ with probabilities $P\left(x_{1}\right), P\left(x_{2}\right), \ldots, P\left(x_{16}\right)$, respectively. The events of System I are defined by the matrix $R^{I}=\left(r_{i j}^{I}\right)$, where $r_{i j}^{I}=1$, if $x_{i} \in A_{j}$; otherwise $r_{i j}^{I}=0$. The events of System II are defined by the matrix $R^{I I}$, similarly. (The matrices $R^{I}$ and $R^{I I}$ were randomly 
generated with different densities.)

$$
R^{I}=\left(\begin{array}{llllllllllll}
0 & 0 & 0 & 0 & 0 & 0 & 1 & 0 & 0 & 0 & 0 & 1 \\
0 & 0 & 1 & 0 & 1 & 0 & 0 & 0 & 0 & 0 & 1 & 0 \\
0 & 0 & 1 & 0 & 0 & 0 & 0 & 0 & 0 & 0 & 0 & 0 \\
0 & 1 & 0 & 0 & 1 & 1 & 0 & 0 & 0 & 0 & 0 & 0 \\
1 & 1 & 0 & 1 & 0 & 0 & 0 & 1 & 0 & 1 & 0 & 0 \\
0 & 1 & 0 & 0 & 1 & 1 & 0 & 1 & 0 & 0 & 0 & 1 \\
0 & 0 & 1 & 1 & 0 & 0 & 0 & 0 & 0 & 0 & 0 & 0 \\
0 & 0 & 0 & 0 & 1 & 0 & 0 & 0 & 0 & 0 & 0 & 0 \\
1 & 0 & 0 & 0 & 0 & 0 & 0 & 0 & 0 & 1 & 1 & 0 \\
1 & 0 & 0 & 0 & 0 & 1 & 0 & 0 & 1 & 1 & 0 & 1 \\
0 & 0 & 1 & 0 & 0 & 1 & 0 & 0 & 0 & 0 & 1 & 0 \\
0 & 0 & 1 & 1 & 0 & 1 & 1 & 0 & 0 & 0 & 0 & 1 \\
0 & 1 & 0 & 0 & 0 & 1 & 1 & 0 & 0 & 1 & 1 & 0 \\
1 & 0 & 0 & 0 & 1 & 0 & 0 & 1 & 0 & 0 & 1 & 0 \\
0 & 1 & 0 & 0 & 0 & 1 & 0 & 0 & 0 & 1 & 0 & 0 \\
0 & 0 & 0 & 0 & 0 & 0 & 0 & 0 & 0 & 0 & 0 & 0
\end{array}\right), R^{I I}=\left(\begin{array}{llllllllllll}
1 & 1 & 0 & 1 & 1 & 1 & 0 & 1 & 1 & 1 & 1 & 0 \\
0 & 1 & 1 & 0 & 1 & 1 & 0 & 0 & 1 & 0 & 1 & 0 \\
1 & 0 & 1 & 1 & 0 & 1 & 1 & 1 & 1 & 1 & 1 & 0 \\
1 & 1 & 1 & 0 & 1 & 1 & 1 & 0 & 1 & 1 & 1 & 1 \\
1 & 1 & 1 & 1 & 1 & 1 & 0 & 1 & 1 & 1 & 1 & 1 \\
1 & 1 & 1 & 1 & 1 & 1 & 1 & 1 & 0 & 0 & 1 & 1 \\
1 & 1 & 1 & 1 & 1 & 1 & 1 & 1 & 1 & 1 & 1 & 1 \\
1 & 1 & 1 & 0 & 0 & 1 & 1 & 1 & 0 & 1 & 1 & 1 \\
1 & 1 & 1 & 1 & 1 & 1 & 1 & 1 & 1 & 1 & 1 & 1 \\
1 & 1 & 1 & 1 & 1 & 1 & 0 & 1 & 0 & 1 & 1 & 1 \\
1 & 1 & 1 & 1 & 1 & 0 & 1 & 1 & 1 & 1 & 0 & 1 \\
1 & 1 & 0 & 1 & 0 & 1 & 0 & 1 & 0 & 1 & 1 & 1 \\
1 & 1 & 1 & 1 & 1 & 1 & 1 & 1 & 1 & 1 & 1 & 1 \\
1 & 1 & 1 & 0 & 1 & 1 & 1 & 1 & 1 & 1 & 1 & 1 \\
1 & 1 & 1 & 1 & 1 & 0 & 0 & 1 & 0 & 1 & 1 & 0 \\
0 & 0 & 0 & 0 & 0 & 0 & 0 & 0 & 0 & 0 & 0 & 0
\end{array}\right)
$$

It is easy to see that at least one event occurs at each outcome except $x_{16}$. From this

$$
P\left(\cup A_{i}\right)=1-P\left(x_{16}\right) .
$$

Regarding the probability of the outcomes we consider the following three cases.

\begin{tabular}{lcccccccc}
\hline & $P\left(x_{1}\right)$ & $P\left(x_{2}\right)$ & $P\left(x_{3}\right)$ & $P\left(x_{4}\right)$ & $P\left(x_{5}\right)$ & $P\left(x_{6}\right)$ & $P\left(x_{7}\right)$ & $P\left(x_{8}\right)$ \\
\hline Case 1: & 0.023 & 0.034 & 0.045 & 0.056 & 0.067 & 0.078 & 0.067 & 0.056 \\
\hline Case 2: & 0.012 & 0.022 & 0.023 & 0.033 & 0.034 & 0.044 & 0.045 & 0.055 \\
\hline Case 3: & 0.0329 & 0.1076 & 0.0599 & 0.1108 & 0.042 & 0.0055 & 0.0508 & 0.1142 \\
\hline
\end{tabular}

\begin{tabular}{ccccccccc}
\hline & $P\left(x_{9}\right)$ & $P\left(x_{10}\right)$ & $P\left(x_{11}\right)$ & $P\left(x_{12}\right)$ & $P\left(x_{13}\right)$ & $P\left(x_{14}\right)$ & $P\left(x_{15}\right)$ & $P\left(x_{16}\right)$ \\
\hline Case 1: & 0.045 & 0.038 & 0.011 & 0.022 & 0.033 & 0.044 & 0.055 & 0.326 \\
\hline Case 2: & 0.056 & 0.066 & 0.067 & 0.077 & 0.078 & 0.088 & 0.089 & 0.211 \\
\hline Case 3: & 0.048 & 0.0235 & 0.0676 & 0.0295 & 0.0441 & 0.1265 & 0.1058 & 0.0313 \\
\hline
\end{tabular}

We use several bounding techniques depending on the known information about the systems. On one hand, we give the (sharp) upper bounds of the univariate binomial moment problem of (1.4) with the first function $f$ in (1.5), based on the information on $S_{1}, S_{2}, S_{3}$. We use the dual method of Prékopa [27].

On the other hand, we subdivide the sequence of $\left\{A_{1}, A_{2}, \ldots, A_{12}\right\}$ into subsequences

$$
\left\{A_{1}, A_{2}, \ldots, A_{6}\right\},\left\{A_{7}, A_{8}, \ldots, A_{12}\right\}
$$

and then into subsequences

$$
\left\{A_{1}, A_{2}, A_{3}, A_{4}\right\},\left\{A_{5}, A_{6}, A_{7}, A_{8}\right\},\left\{A_{9}, A_{10}, A_{11}, A_{12}\right\}
$$

and then into

$$
\left\{A_{1}, A_{2}, A_{3}\right\},\left\{A_{4}, A_{5}, A_{6}\right\},\left\{A_{7}, A_{8}, A_{9}\right\},\left\{A_{10}, A_{11}, A_{12}\right\} .
$$

We consider the multivariate binomial moment problem (1.11) with the function $f$ in (1.12) with the appropriate sets $H$ (1.14), respectively. Prékopa [30] has shown that the even (odd) order divided differences of $f$ in (1.12) are nonpositive (nonnegative). This means that in cases where $m+1$ and $m_{j}+1, j=1, \ldots, s$ (where $s$ is the number of subsequences), are even we can give upper bounds by the application of the Min 
TABLE 1

Results of System I.

\begin{tabular}{lccccc}
\hline & Univariate & $(5.5) m=3$, & $(5.6) m=3$, & $(5.7) m=3$, & $m=3$ \\
& $S_{1}, S_{2}, S_{3}$ & $\begin{array}{c}m_{1}=3, \\
m_{2}=3\end{array}$ & $\begin{array}{c}m_{1}=3, \\
m_{2}=3, \\
m_{3}=3\end{array}$ & $\begin{array}{c}m_{1}=m_{2}=3, \\
m_{3}=m_{4}=3\end{array}$ & bound \\
& & & 0.969 & 0.697 & 0.785 \\
\hline Case 1: & 0.7412 & 1 & 1 & 0.801 & 0.900 \\
\hline Case 2: & 0.8595 & 1 & 1 & 1 & 1 \\
\hline Case 3: & 1 & 1 & & 1 & \\
\hline
\end{tabular}

TABLE 2

Results of System II.

\begin{tabular}{lccccc}
\hline & Univariate & $(5.5) m=3$, & $(5.6) m=3$, & $(5.7) m=3$, & $m=$ \\
& $S_{1}, S_{2}, S_{3}$ & $\begin{array}{c}m_{1}=3, \\
m_{2}=3\end{array}$ & $\begin{array}{c}m_{1}=3, \\
m_{2}=3, \\
m_{3}=3\end{array}$ & $\begin{array}{c}m_{1}=m_{2}=3, \\
m_{3}=m_{4}=3\end{array}$ & $\begin{array}{c}\text { bound } \\
\text { bound }\end{array}$ \\
\hline Case 1: & 0.781345 & 0.749467 & 0.702333 & 0.674 & 0.685 \\
\hline Case 2: & 0.921273 & 0.873867 & 0.807333 & 0.789 & 0.789 \\
\hline Case 3: & 1 & 1 & 1 & 0.9687 & 0.9687 \\
\hline
\end{tabular}

Algorithm for $-f(\boldsymbol{z})$. In this example we use the Min Algorithm with parameters $m=3, m_{j}=3, j=1, \ldots, s$.

Finally, we give the so-called cherry tree bounds of Bukszár and Prékopa [4]. These bounds are based on the knowledge of the individual probabilities of the occurrences of the events, of the intersections of pairs of events, and of the intersections of three events. These bounds are always at least as good as the Hunter-Worsley second order bounds (see Hunter [17]).

The results for System I and System II are summarized in Tables 1 and 2, respectively. Comparing the bounds, we can see that we get the best bound in case of the subsequences (5.7).

Regarding the order of the columns in Tables 1 and 2, we took more and more information on the system into account; i.e., in case of the univariate binomial moments we just considered the sums of probabilities of events, intersections of pairs of events, and triples of events. In the case of multivariate binomial moments we considered the sums of those probabilities of smaller groups of the events; i.e., we separated the sums of the univariate moments into subsums. Finally, in the case of cherry trees we used individual probabilities. This would imply that we should get better and better bounds. However, the bounds of our paper as well as the cherry tree bounds are not sharp; that is why it could happen that the Min Algorithm gave better bounds, using less information, than the cherry tree bounds of Bukszár and Prékopa [4].

The numerical results confirm that our method yields a new effective tool for bounding the probability of the union of events based on the knowledge of probabilities of the intersection up to three events. On the other hand, we can see that the bivariate bounds corresponding to the subsequences (5.5) are much weaker than the bounds corresponding to the binomial MDMPs of higher dimensions. Since our generalization of the bivariate Min Algorithm gives the possibility of taking more detailed information into account, it can give substantially better bounds. 
Example 5.3. We shall also give upper bounds for the probability of the union of events. There are 20 events $A_{1}, A_{2}, \ldots, A_{20}$, and 16 outcomes, $x_{1}, x_{2}, \ldots, x_{16}$ with probabilities $P\left(x_{1}\right), P\left(x_{2}\right), \ldots, P\left(x_{16}\right)$, respectively. The events of the system are defined by the matrix $R=\left(r_{i j}\right)$, where $r_{i j}=1$, if $x_{i} \in A_{j}$; otherwise $r_{i j}=0$.

$$
R=\left(\begin{array}{llllllllllllllllllll}
1 & 0 & 0 & 0 & 1 & 0 & 0 & 0 & 0 & 0 & 0 & 1 & 0 & 0 & 1 & 0 & 0 & 1 & 0 & 1 \\
0 & 1 & 0 & 1 & 0 & 0 & 1 & 0 & 0 & 1 & 0 & 0 & 0 & 1 & 0 & 0 & 0 & 1 & 0 & 0 \\
1 & 0 & 1 & 0 & 1 & 0 & 0 & 1 & 0 & 0 & 0 & 0 & 0 & 0 & 0 & 1 & 0 & 0 & 1 & 0 \\
0 & 1 & 0 & 0 & 0 & 1 & 0 & 0 & 1 & 0 & 0 & 1 & 0 & 0 & 1 & 0 & 0 & 1 & 0 & 0 \\
1 & 0 & 1 & 0 & 1 & 0 & 0 & 1 & 0 & 0 & 1 & 0 & 0 & 1 & 0 & 0 & 1 & 0 & 1 & 0 \\
0 & 1 & 0 & 0 & 1 & 0 & 1 & 0 & 1 & 0 & 1 & 1 & 0 & 0 & 1 & 0 & 1 & 0 & 0 & 1 \\
1 & 0 & 0 & 1 & 0 & 1 & 0 & 1 & 0 & 0 & 1 & 0 & 1 & 0 & 0 & 1 & 0 & 0 & 0 & 0 \\
0 & 1 & 0 & 1 & 0 & 0 & 0 & 0 & 1 & 0 & 1 & 0 & 0 & 0 & 0 & 1 & 0 & 1 & 0 & 1 \\
1 & 0 & 1 & 0 & 0 & 1 & 0 & 1 & 0 & 1 & 0 & 1 & 0 & 1 & 0 & 0 & 0 & 1 & 0 & 1 \\
1 & 0 & 0 & 1 & 0 & 0 & 1 & 0 & 0 & 1 & 0 & 0 & 1 & 0 & 0 & 0 & 1 & 0 & 0 & 0 \\
0 & 1 & 0 & 0 & 1 & 0 & 1 & 0 & 1 & 0 & 0 & 1 & 0 & 1 & 0 & 1 & 0 & 1 & 0 & 1 \\
0 & 0 & 0 & 1 & 0 & 0 & 0 & 1 & 0 & 0 & 1 & 0 & 0 & 1 & 0 & 0 & 1 & 0 & 0 & 0 \\
1 & 0 & 0 & 1 & 0 & 1 & 0 & 0 & 0 & 1 & 0 & 0 & 1 & 0 & 1 & 0 & 0 & 1 & 0 & 1 \\
0 & 1 & 0 & 0 & 0 & 1 & 0 & 0 & 1 & 0 & 0 & 0 & 1 & 0 & 0 & 1 & 0 & 0 & 1 & 0 \\
0 & 0 & 0 & 1 & 0 & 0 & 0 & 0 & 0 & 0 & 0 & 0 & 0 & 0 & 0 & 0 & 0 & 0 & 0 & 1 \\
0 & 0 & 0 & 0 & 0 & 0 & 0 & 0 & 0 & 0 & 0 & 0 & 0 & 0 & 0 & 0 & 0 & 0 & 0 & 0
\end{array}\right)
$$

The matrix $R$ is taken from System 1 of Example 2 in Prékopa and Gao [34], but we use the probabilities of outcomes of (5.4) instead of their probabilities because in their system the probability of the union is nearly 1 . We consider the univariate binomial DMP for $S_{1}, S_{2}, S_{3}$ and for $S_{1}, \ldots, S_{5}$. We also give the results of the multivariate binomial DMP (1.11) with the function $f$ in (1.12) with the appropriate sets $H$ (1.14). We consider the subdivisions

$$
\left\{A_{1}, \ldots, A_{7}\right\},\left\{A_{8}, \ldots, A_{14}\right\},\left\{A_{15}, \ldots, A_{20}\right\},
$$

with $m=m_{j}=5(j=1,2,3)$, and

$$
\left\{A_{1}, \ldots, A_{5}\right\},\left\{A_{6}, \ldots, A_{10}\right\},\left\{A_{11}, \ldots, A_{15}\right\},\left\{A_{16}, \ldots, A_{20}\right\},
$$

with $m=m_{j}=5(j=1,2,3,4)$. (The MDMPs with third order binomial moments yield trivial bounds.) We also give the cherry tree bounds. The results are summarized in Table 3. Among the third order bounds once the univariate binomial DMP bound, once the cherry tree bound was the best, however, none of them were useful. However, using moments up to the order 5 our binomial MDMP, with subdivision (5.10), yields the best (in these cases the sharp) bounds.

TABLE 3

Results of the system of Example 5.3.

\begin{tabular}{lccccc}
\hline & Univariate & Cherry tree & Univariate & $(5.9) m=5$, & $(5.10) m=5$, \\
& $S_{1}, S_{2}, S_{3}$ & bound & $S_{1}, \ldots, S_{5}$ & $m_{j}=5$ & $m_{j}=5$ \\
\hline Case 1: & 0.794036 & 0.823 & 0.6882 & 0.96691 & 0.674 \\
\hline Case 2: & 0.960964 & 0.901 & 0.810878 & 1 & 0.789 \\
\hline Case 3: & 1 & 1 & 0.99522 & 1 & 0.9687 \\
\hline
\end{tabular}




\section{REFERENCES}

[1] N. I. Akhiezer, The Classical Moment Problem and Some Related Questions in Analysis, Hafner Publishing, New York, 1965.

[2] I. Bienaymé, Considérations a l'appui de la découverte de Laplace sur la loi de probabilité dans la méthode des moindres carré, C.R. Acad. Sci. Paris, 37 (1853), pp. 309-326.

[3] E. Boros And A. PrÉKopa, Closed form two-sided bounds for probabilities that at least $r$ and exactly $r$ out of $n$ events occur, Math. Oper. Res., 14 (1989), pp. 317-342.

[4] J. Bukszár and A. PrÉKopa, Probability bounds with cherry trees, Math. Oper. Res., 26 (2001), pp. 174-192.

[5] J. Bukszár and T. SzÁntai, Probability bounds given by hypercherry trees, Optim. Methods Softw., 17 (2002), pp. 409-422.

[6] P. Chebyshev, Sur les valeurs limites des intégrales, Journal de Mathématiques Pures et Appliquées, 19 (1874), pp. 157-160.

[7] P. Chebyshev, Sur deux théorèmes relatifs aux probabilités, Acta Math., 14 (1890), pp. 305315.

[8] C. I. FÁBIÁN AND Z. SzŐKE, Solving two-stage stochastic programming problems with level decomposition, Comput. Manag. Sci., 4 (2007), pp. 313-353.

[9] M. Gasca And T. SAuer, Polynomial interpolation in several variables, Adv. Comput. Math., 12 (2000), pp. 377-410.

[10] M. GasCa AND T. SAUER, On the history of multivariate polynomial interpolation, J. Comput. Appl. Math., 122 (2000), pp. 23-35.

[11] A. HABIB And T. SzÁNTAI, New bounds on the reliability of the consecutive k-out-of-r-from-n: F system, Reliability Engineering and System Safety, 68 (2000), pp. 97-106.

[12] H. Hamburger, Beiträge zur Konvergenztheorie der Stieltjesschen Kettenbrüche, Math. Z., 4 (1919), pp. 186-222.

[13] H. Hamburger, Uber eine Erweiterung des Stieltjesschen Momentproblems, Math. Ann., 81 (1920), pp. 235-319; 82 (1921), pp. 120-164 and pp. 168-187.

[14] F. HAusdorfF, Summationsmethoden und Momentfolgen, I and II, Math. Z., 16 (1921), pp. 74109 and pp. 280-299.

[15] F. Hausdorff, Momentprobleme für ein endliches Intervall, Math. Z., 16 (1923), pp. 220-248.

[16] X. Hou AND A. PrÉKOPA, Monge property and bounding multivariate probability distribution functions with given marginals and covariances, SIAM J. Optim., 18 (2007), pp. 138-155.

[17] D. Hunter, An upper bound for the probability of a union, J. Appl. Prob., 13 (1976), pp. 597603.

[18] T. H. KJeldsen, The early history of the moment problem, Historia Math., 20 (1993), pp. 1944.

[19] M. G. Krein and A. A. Nudelman, The Markov Moment Problem and Extremal Problems. Translations of Mathematical Monographs 50, AMS, Providence, RI, 1977.

[20] G. MÁDI-NAGY, A method to find the best bounds in a multivariate discrete moment problem if the basis structure is given, Studia Sci. Math. Hungar., 42 (2005), pp. 207-226.

[21] G. MÁdi-NAGY And A. PrÉKOPA, On multivariate discrete moment problems and their applications to bounding expectations and probabilities, Math. Oper. Res., 29 (2004), pp. 229-258.

[22] G. MÁdi-NAGy AND A. PrÉKOPA, Bounding Expectations of Functions of Random Vectors with Given Marginals and Some Moments: Applications of the Multivariate Discrete Moment Problem, RUTCOR Research Report, 11-2007, Piscataway, NJ, 2007.

[23] A. Markov, On Certain Applications of Algebraic Continued Fractions, Ph.D. thesis, St. Petersburg, Russia, 1884.

[24] T. Popoviciu, Les Fonctions Convexes, Actualités Scientifiques et Industrielles 992, Hermann et Cie, Paris, 1944.

[25] A. PrÉKopa, Boole-Bonferroni inequalities and linear programming, Oper. Res., 36 (1988), pp. 145-162.

[26] A. PRÉKOPA, Sharp bounds on probabilities using linear programming, Oper. Res., 38 (1990), pp. 227-239.

[27] A. PRÉKOPA, The discrete moment problem and linear programming, Discrete Appl. Math., 27 (1990), pp. 235-254.

[28] A. PrÉKOPA, Inequalities on expectations based on the knowledge of multivariate moments, in Stochastic Inequalities, M. Shaked and Y. L. Tong, eds., Lecture Notes Monogr. Ser. 22, Inst. Math. Statist., Hayward, CA, 1992, pp. 309-331.

[29] A PrÉKopa, Stochastic Programming, Kluwer Academic Publishers, Dordrecht, The Netherlands, 1995.

[30] A. PRÉKOPA, Bounds on probabilities and expectations using multivariate moments of discrete distributions, Studia Sci. Math. Hungar., 34 (1998), pp. 349-378. 
[31] A. PrÉKopa, The use of discrete moment bounds in probabilistic constrained stochastic programming models, Ann. Oper. Res., 85 (1999), pp. 21-38.

[32] A. PrÉKopa, On Multivariate Discrete Higher Order Convex Functions and Their Applications, RUTCOR Research Report, 39-2000, Piscataway, NJ, 2000. Also in, Proceedings of the Sixth International Conference on Generalized Convexity and Monotonicity, Karlovasi, Samos, Greece, to appear.

[33] A. Prékopa and G. Alexe, Dual Methods for the Numerical Solution of the Univariate Power Moment Problem, RUTCOR Research Report, 14-2003, Piscataway, NJ, 2003.

[34] A. PrÉKopa AND L. GaO, Bounding the probability of the union of events by aggregation and disaggregation in linear programs, Discrete Appl. Math., 145 (2005), pp. 444-454.

[35] A. PrÉKOPA And G. Mádi-NAGY, A class of multiattribute utility functions, Econom. Theory, 34 (2008), pp. 591-602.

[36] F. Riesz, Sur le probléme des moments, Arkiv for Matematik Astronomi och Fysik, 17 (1923), pp. $1-52$.

[37] S. M. Samuels and W. J. Studden, Bonferroni-type probability bounds as an application of the theory of Tchebycheff system, in Probability, Statistics and Mathematics, Papers in Honor of Samuel Karlin, Academic Press, Boston, 1989, pp. 271-289.

[38] T. J. Stieltues, Recherches sur les fractions continues, Ann. Fac. Sci. Univ. Toulouse, 8 (1894), pp. J76-J122; 9 (1895), pp. A5-A47.

[39] T. SzÁNTAI, Evaluation of a special multivariate gamma distribution function, Math. Programming Stud., 27 (1986), pp. 1-16.

[40] T. SzÁNTAI, Improved bounds and simulation procedures on the value of the multivariate normal probability distribution function, Ann. Oper. Res., 100 (2000), pp. 85-101. 